\title{
Universal features of gravitational waves emitted by superkick binary black hole systems
}

\author{
Sizheng Ma๑, ${ }^{1, *}$ Matthew Giesler $\odot,{ }^{2}$ Vijay Varma $\odot,{ }^{3,2}$ Mark A. Scheel, ${ }^{1}$ and Yanbei Chen ${ }^{1, \dagger}$ \\ ${ }^{1}$ TAPIR 350-17, California Institute of Technology, 1200 E California Boulevard, \\ Pasadena, California 91125, USA \\ ${ }^{2}$ Cornell Center for Astrophysics and Planetary Science, Cornell University, Ithaca, New York 14853, USA \\ ${ }^{3}$ Department of Physics, Cornell University, Ithaca, New York 14853, USA
}

(Received 10 July 2021; accepted 9 August 2021; published 1 October 2021)

\begin{abstract}
We use numerical relativity to study the merger and ringdown stages of "superkick" binary black hole systems (those with equal mass and antiparallel spins). We find a universal way to describe the mass and current quadrupole gravitational waves emitted by these systems during the merger and ringdown stage: (i) The time evolutions of these waves are insensitive to the progenitor's parameters (spins) after being normalized by their own peak values. (ii) The peak values, which encode all the spin information of the progenitor, can be consistently fitted to formulas inspired by post-Newtonian theory. We find that the universal evolution of the mass quadrupole wave can be accurately modeled by the so-called Backwards One-Body (BOB) model. However, the BOB model, in its present form, leads to a lower waveform match and a significant parameter-estimation bias for the current quadrupole wave. We also decompose the ringdown signal into seven overtones, and study the dependence of mode amplitudes on the progenitor's parameters. Such dependence is found to be insensitive to the overtone index (up to a scaling factor). Finally, we use the Fisher matrix technique to investigate how the ringdown waveform can be at least as important for parameter estimation as the inspiral stage. Assuming the Cosmic Explorer, we find the contribution of ringdown portion dominates as the total mass exceeds $\sim 250 M_{\odot}$. For massive binary black hole $(\mathrm{BBH})$ systems, the accuracy of parameter measurement is improved by incorporating the information of ringdown - the ringdown sector gives rise to a different parameter correlation from inspiral stage; hence, the overall parameter correlation is reduced in full signal.
\end{abstract}

DOI: 10.1103/PhysRevD.104.084003

\section{INTRODUCTION}

The recently detected gravitational wave (GW) signal, GW190521, is consistent with the merger of two black holes (BHs) with masses of $85 M_{\odot}$ and $66 M_{\odot}[1,2]$. The detection of this event, together with its candidate optical counterpart ZTF19abanrhr [3], indicates the potential existence of BHs in the mass gap predicted by (pulsational) pair-instability supernova theory $[2,4,5]$. A few studies also suggest that this system could admit an extremely eccentric $[2,6]$, hyperbolic [7], or a head-on [2,8] merger interpretation, placing possible constraints on the binary's formation channel $[6,7,9]$. For such an event, most of the GW detected by the Advanced LIGO [10], VIRGO [11], and KAGRA $[12,13]$ network is dominated by the merger and ringdown portions. This demonstrates the importance of understanding ringdowns for detecting more GW190521like cases in the near future [14].

sma@caltech.edu

†yanbei@caltech.edu
The ringdown signal can be treated as a superposition of damped sinusoids, corresponding to the quasinormal modes (QNMs) of the final BH [15]. Due to the no-hair theorem [16], the QNM frequencies and damping time for a spinning BH in general relativity (GR) are fully determined by its mass and angular momentum. Therefore, measuring a QNM from a GW event can allow us to determine the properties of the final $\mathrm{BH}$. Alternatively, if multiple modes are observed at the same time, we can use them to test the no-hair theorem and general relativity [17-28,28-36], and also constrain modified gravity [32,33,37-39].

In addition to measuring QNM frequencies, extensive studies have also been carried out to explore the relationship between progenitor's parameters and additional ringdown signatures. For instance, the spin (magnitude and direction) and mass of the remnant BH were fitted to progenitor's spins $\left(\chi_{1,2}\right)$ and mass ratio $\left(q=m_{\text {heavy }} / m_{\text {light }}>1\right)$ [40-55], as well as the peak amplitude of GW strain [56], using numerical relativity (NR) [36], the effective-one-body (EOB) approach [57], and also a hybrid way that involves multitimescale post-Newtonian integrations and numericalrelativity surrogate models [58]. The gravitational wave 
frequency at peak amplitude [53,59] and the peak GW luminosity $[50,53,60]$ were both found to have a clean dependence on the progenitor's parameters. The above facts clearly imply that the initial conditions (e.g., at merger) for the progenitor are encoded in the ringdown portion of $\mathrm{GW}$, including QNM frequencies and amplitudes. Therefore it is not surprising that the ringdown can be used to learn about the component properties.

Apart from conveying the importance of ringdown studies, the detection of the candidate optical counterpart of GW190521 has also provided us with a new scheme to measure the gravitational recoil [3]. General relativity predicts that a system is kicked after merger due to the linear momentum carried away by GW [61-63]. By applying various methods, including NR, post-Newtonian (PN) theory [64-66], EOB [67], and the close-limit approximation [68], several studies showed that the kick velocity is a result of the asymmetry between different GW modes [69], or alternatively, the beating between the mass and current quadrupole waves [70,71], caused by the unequal mass [72-76] and spins [75-83]. In particular, the superkick (SK) $[78,80,84-86]$ configurations lead to relatively large kick velocities. In our paper, we adopt two types of SK configurations: SKu and SKd, whose sketches are shown in Fig. 1. Both systems have equal mass, spin magnitude, and tilt angles. As for SKd, two individual spins are antiparallel, whereas, for SKu, only the spin components in the orbital plane are opposite. SKu and SKd are fully characterized by $\left(\chi_{\text {init }}, \phi_{\text {init }}, \theta_{\text {init }}\right)$, where the subscript refers to a reference time in the inspiral regime: $\chi_{\text {init }}$ is the magnitude of the dimensionless spin; $\theta_{\text {init }}$ is the polar angle of one of the holes (relative to the orbital angular momentum $\vec{L}$ ); $\phi_{\text {init }}$ is the azimuthal angle between the in-(orbital)plane spin and the separation vector pointing from the lighter to the heavier $\mathrm{BH}$.

During the evolution, the effect of frame-dragging from two antiparallel in-plane spins moves the center of mass up and down in the inertial frame [87]. This process is halted as the common horizon forms [88-90], and the kick is imparted. In addition, the SKu system usually emits more energy and linear angular momentum than SKd because of the orbital hang-up effect, which arises due to the need to radiate way additional angular momentum before the binary can merge [84], and leads to a larger kick. Recently simulations showed that the kick for the SKu system could be as large as $5000 \mathrm{~km} / \mathrm{s}$ (if extrapolated to the maximal spin) $[85,86]$. Such a large kick will lead to important astrophysical consequences [91-94], as well as Doppler shifts in GWs [95], which could be detected by current and future detectors [96,97]. Numerous studies have been implemented to fit kick velocities to progenitor's spins and mass ratio $[40,49,50,53,79,80,98]$. In particular, the development of numerical relativity surrogate model $[54,55,99-102]$ has allowed a systematic study to explore much larger parameter space [103].

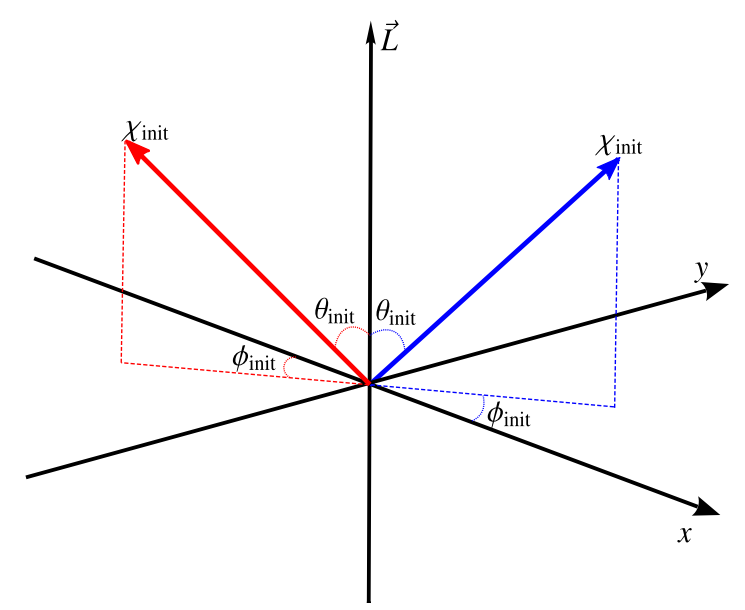

(a) $\mathrm{SKu}$

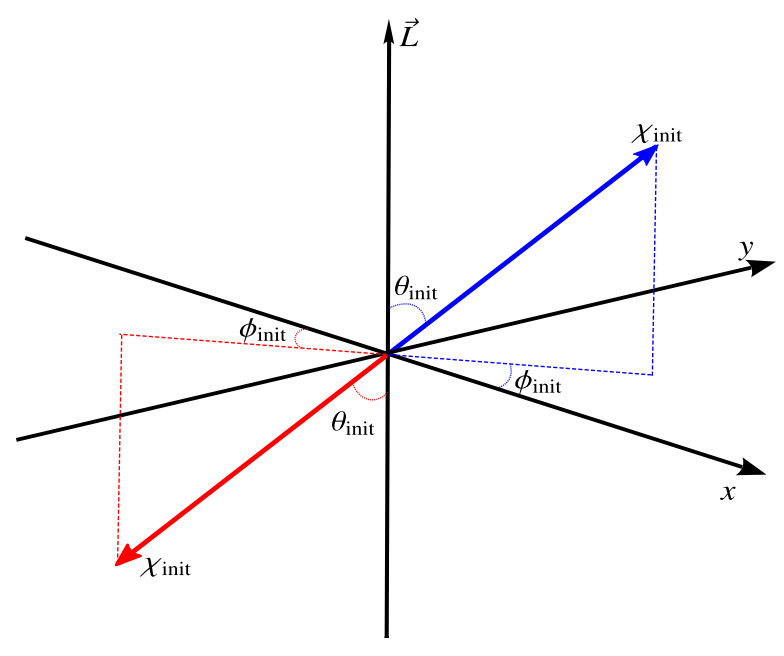

(b) SKd

FIG. 1. Sketches for a SKu (a) and a SKd (b) system. Two arrows (in different colors) represent two individual spins. The letter " $u$ " and "d" refer to the up- and down-state for the red arrow. Both SKu and SKd systems have equal mass BHs with the same dimensionless spin magnitude $\chi_{\text {init }}$. For SKd, two individual spins are antiparallel, whereas, for $\mathrm{SKu}$, only the orbital-plane components are opposite. SKd and SKu are fully characterized by three parameters: $\left(\chi_{\text {init }}, \theta_{\text {init }}, \phi_{\text {init }}\right)$, where $\theta_{\text {init }}$ stands for the polar angle of one of the holes (relative to the orbital angular momentum), and $\phi_{\text {init }}$ the azimuthal angle of the in-plane spin measured from the line of two BHs. Three parameters are specified at a reference time in the inspiral regime (labeled by the subscript "init").

Interestingly, GW190521 was found to be consistent with a large in-plane spin configuration. Its kick posterior is much broader and is consistent with $0-3500 \mathrm{~km} / \mathrm{s}$ [2]. Meanwhile, its potential optical counterpart was predicted to have a kick velocity of $\sim 200 \mathrm{~km}$ [3]. In the future, it is still likely to detect GW events with non-negligible 
TABLE I. A summary of 12 of our NR simulations with SpEC. All systems are in the SKd configuration, with the individual dimensionless $\operatorname{spin} \chi_{\text {init }}=0.4, \theta_{\text {init }}=\pi / 2$, and $\phi_{\text {init }} \in[-\pi, \pi]$. The reference (initial) orbital frequency is chosen to be 0.0175 (in the unit of total mass). The first and second columns are the name of runs used in this paper, while the third column corresponds to the name in the Simulating eXtreme Spacetimes Collaboration catalog. The fourth column gives $\phi_{\text {init }}$. The last three columns correspond to the mass, kick velocity, and spin of the final BH. A summary of SKu configurations is in Table V.

\begin{tabular}{|c|c|c|c|c|c|c|}
\hline \multicolumn{3}{|c|}{ Run label } & \multirow[b]{2}{*}{$\phi_{\text {init }}(\mathrm{rad})$} & \multirow[b]{2}{*}{$m_{f} / M$} & \multirow[b]{2}{*}{$v_{f}^{z}\left(\times 10^{-3}\right)$} & \multirow[b]{2}{*}{$\chi_{f}$} \\
\hline & This paper & SXS:BBH & & & & \\
\hline \multirow[t]{12}{*}{$\mathrm{SKd} 4$} & "01" & 2451 & 2.25 & 0.952 & 2.36 & 0.686 \\
\hline & “02” & 2452 & -3.04 & 0.951 & -4.73 & 0.684 \\
\hline & “03” & 2453 & -1.70 & 0.951 & 1.71 & 0.685 \\
\hline & “04” & 2454 & 0.66 & 0.951 & -4.45 & 0.683 \\
\hline & “05” & 2455 & 1.30 & 0.951 & -1.68 & 0.685 \\
\hline & "06" & 2456 & 2.88 & 0.951 & 4.75 & 0.684 \\
\hline & “07” & 2457 & -2.58 & 0.951 & 4.47 & 0.683 \\
\hline & "08" & 2458 & -1.07 & 0.952 & -2.11 & 0.686 \\
\hline & "09" & 2459 & -2.93 & 0.951 & 4.94 & 0.683 \\
\hline & "10" & 2460 & -1.78 & 0.951 & 1.24 & 0.686 \\
\hline & "11" & 2461 & -1.36 & 0.952 & -1.41 & 0.686 \\
\hline & $" 12 "$ & 2462 & 0.21 & 0.951 & -4.93 & 0.683 \\
\hline
\end{tabular}

gravitational recoils, and even SK-like binaries [104]. Accordingly, in this paper, we aim to explore the features of ringdown for SKd binaries carefully and relate them to the phenomenon of gravitational recoil. Specifically, we shall focus on the amplitudes of QNMs [105-107], as well as mass and current quadrupole waves [70], and study how those features depend on the progenitor's parameters. Comparing to a generic BBH system, a SKd system has several advantages that can ease the difficulty of analysis. (i) The parameter space for a SKd binary is $3 \mathrm{D}$, i.e., $\left(\chi_{\text {init }}, \phi_{\text {init }}, \theta_{\text {init }}\right)$, instead of generally 7D. (ii) SKd configurations have a high level of symmetry. Subsequently, the orbital angular momentum is nonprecessing, and the spin direction of the remnant $\mathrm{BH}$ is fixed during the merger. This allows us to conveniently choose coordinates in which only the $(2,2)$ and $(2,-2)$ modes dominate. (iii) The mass and spin of the remnant $\mathrm{BH}$ are not impacted by varying $\left(\chi_{\text {init }}, \phi_{\text {init }}, \theta_{\text {init }}\right)$, nor are the QNM frequencies. Hence we can study the mode excitation (complex) amplitudes exclusively while avoiding changes in the mode frequencies.

In our study, we use waveforms generated by the Spectral Einstein Code (SpEC) [108], and two NR surrogate models, also based on SpEC: NRSur7dq4, NRSur7dq4Remnant $[54,55]$. In particular, NRSur7dq4 is a waveform model valid for mass ratio $<4$ and dimensionless spin magnitudes $<0.8$, while NRSur7dq4Remnant is a model that predicts the mass, spin, and kick velocity of the remnant $\mathrm{BH}$ from the parameter of individual merging BHs. Meanwhile, we have in total $35 \mathrm{NR}$ simulations where systems are either in the SKd (Table I) or the SKu (Table V) configuration. The dimensionless spin of $\mathrm{BH}$ ranges from 0.4 to 0.95 . Those runs will be available in the Simulating eXtreme Spacetimes (SXS) Collaboration catalog [109,110].
We have checked that our NR runs agree with the predictions of NRSur7dq4, with mismatches $\sim 10^{-5}-10^{-4}$. For each simulation, we evolve with three numerical resolutions. Among those cases, the largest kick is $\sim 4050 \mathrm{~km}$ (Table V).

This paper is organized as follows. In Sec. II, we decompose ringdown into QNMs (7 overtones) and explore the dependence of mode amplitudes on the progenitor's parameters. In Sec. III, we study the phenomenon of radiative mass and current quadrupole waves and relate them to kick velocity. Then in Sec. IV, we apply the backward-one-body (BOB) model, conceived recently by McWilliams [111], to SK binaries. Section V focuses on parameter estimation, where we use the Fisher information matrix formalism to discuss the parameter correlations in the ringdown signal. Finally, in Sec. VI we summarize our results.

Throughout this paper we use the geometric units with $G=c=1$. We use $M$ to refer to the initial total mass of the binary system. All GW waveforms are aligned in the time domain such that $t=0 M$ corresponds to the time of the peak of the total amplitude $\sqrt{\sum_{l, m}\left|h_{l m}\right|^{2}}$.

\section{MULTIPOLE DECOMPOSITION OF THE WAVEFORM AND QUASINORMAL MODE EXCITATIONS}

In this section, we decompose the ringdown signal into QNMs and study how each mode is excited.

\section{A. Multipole decomposition of the waveform}

In a spherical polar coordinate system, with an observer located at the $(l, \beta)$ direction, following the widely used 
convention for defining the + and $\times$ polarizations of the gravitational wave [112], one can define a complex strain

$$
h(t, l, \beta)=h_{+}(t, l, \beta)-i h_{\times}(t, l, \beta),
$$

and further decompose it into a sum over a set of spinweighted spherical harmonics ${ }_{2} Y_{\ell m}(l, \beta)$ :

$$
\begin{aligned}
h(t, l, \beta) & =h_{+}(t, l, \beta)-i h_{\times}(t, l, \beta) \\
& =\sum_{\ell=2}^{\infty} \sum_{m=-\ell}^{\ell} \frac{1}{D} h_{\ell m}(t)_{-2} Y_{\ell m}(l, \beta),
\end{aligned}
$$

where $D$ is the distance between the source and the observer. Meanwhile, it is also natural to group $h_{\ell, m}$ and $h_{\ell,-m}$ into mass and current quadrupole waves [71], writing

$$
\begin{gathered}
I_{\ell m}=\frac{1}{\sqrt{2}}\left[h_{\ell m}+(-1)^{m} h_{\ell,-m}^{*}\right], \\
S_{\ell m}=\frac{i}{\sqrt{2}}\left[h_{\ell m}-(-1)^{m} h_{\ell,-m}^{*}\right] .
\end{gathered}
$$

Here $I_{\ell m}\left(S_{\ell m}\right)$ is the mass (current) quadrupole wave, proportional to the $\ell$-th order time derivative of the mass (current) $\ell$-pole moment. For the SKd configuration, $h_{2, \pm 2}$ always dominates over other modes; hence, we shall primarily focus on these two modes.

\section{B. QNM excitation in multipolar modes}

As discussed in Ref. [30], the ringdown portion of $h_{2, \pm 2}$ of a nonprecessing system can be modeled as a sum of QNMs, as early as $t=0 M$, which is defined as the moment of time at which $\sqrt{\sum_{l, m}\left|h_{l m}\right|^{2}}$ peaks. The expansion reads:

$$
\begin{aligned}
h_{22} & =\sum_{n=0}^{N} \mathcal{A}_{22 n} e^{i \psi_{22 n}} e^{-i \omega_{22 n} t}, \\
h_{2,-2} & =\sum_{n=0}^{N} \mathcal{A}_{2,-2 n} e^{i \psi_{2,-2 n}} e^{i \omega_{22 n}^{*} t}, \quad t \geq 0 M,
\end{aligned}
$$

where $\mathcal{A}_{22 n} e^{i \psi_{22 n}}$ and $\mathcal{A}_{2,-2 n} e^{i \psi_{2,-2 n}}$ are the complex amplitudes of the $n$-th overtone, while $\omega_{22 n}$ and $-\omega_{22 n}^{*}$ are the mode frequencies. Note that $\omega_{22 n}$ and $-\omega_{22 n}^{*}$ have opposite real parts and equal imaginary parts; both correspond to the prograde $\ell=2$ quasinormal mode. In Eq. (4) we have adopted the approximation that the angular wave function of the $(2,2)$ mode is given by the spin-weighted spherical harmonics instead of the spin-weighted spheroidal harmonics-the spheroidal-spherical mixing [113,114] can be ignored because of the moderate spin of final BHs $(\sim 0.68)$ studied in this paper. In this way, both the prograde, $\omega_{22}$, and the retrograde, $\omega_{2,-2}$, modes share the same angular wave function. Meanwhile, the retrograde modes $\omega_{2,-2 n}$ and $-\omega_{2,-2 n}^{*}$ [see Eq. (3.6) of Ref. [107]] are negligible in our case.

Inserting Eqs. (4) to Eqs. (3) we have

$$
\begin{aligned}
I_{22} & =\sum_{n=0}^{N} \mathcal{A}_{n}^{(I)} e^{i \varphi_{n}^{(I)}} e^{-i \omega_{22 n} t}, \\
S_{22} & =\sum_{n=0}^{N} \mathcal{A}_{n}^{(S)} e^{i \varphi_{n}^{(S)}} e^{-i \omega_{22 n} t}, \quad t \geq 0 M,
\end{aligned}
$$

with

$$
\begin{gathered}
\mathcal{A}_{n}^{(I)} e^{i \varphi_{n}^{(I)}}=\frac{1}{\sqrt{2}}\left(\mathcal{A}_{22 n} e^{i \psi_{22 n}}+\mathcal{A}_{2,-2 n} e^{-i \psi_{2,-2 n}}\right), \\
\mathcal{A}_{n}^{(S)} e^{i \varphi_{n}^{(S)}}=\frac{i}{\sqrt{2}}\left(\mathcal{A}_{22 n} e^{i \psi_{22 n}}-\mathcal{A}_{2,-2 n} e^{-i \psi_{2,-2 n}}\right) .
\end{gathered}
$$

To give an example, we fit the ringdown portion of SKd4 set of NR simulations (Table I) with 7 overtones, following the procedure of Ref. [30]. We use unweighted linear least squares to fit the mode amplitudes and use nonlinear least squares to fit the final spin and mass. The mode frequency $\omega_{22 n}$ is obtained from a Python package qnm [115].

First focusing on $I_{22}$ and $S_{22}$, we plot $\mathcal{A}_{n}^{(I)}$ (mass) and $\mathcal{A}_{n}^{(S)}$ (current) as functions of $\phi_{\text {init }}$ in Fig. 2. We can see $\mathcal{A}_{n}^{(I)}>\mathcal{A}_{n}^{(S)}$ for any $n$, and both of them peak at $n=4$. Patterns have a rough period $\pi$. An interesting feature is that the dependence on $\phi_{\text {init }}$ is similar for all overtones (up to a scaling factor). The analogous universal feature for extreme-mass-ratio inspiral (EMRI) was explored by Lim et al. [107]. After a proper normalization [see their Eq. (5.1)], the angular dependence of mode amplitudes is insensitive to the mode indices [see their Fig. 12]. Similarly, for the phase of mode amplitude $\varphi_{n}^{(I)}$ and $\varphi_{n}^{(S)}$, as shown in Fig. 3, their dependence on $\phi_{\text {init }}$ is also insensitive to the overtone index $n$.

The features of $\mathcal{A}_{n}$ and $\varphi_{n}$ allow us to conclude that the dependence of QNM amplitudes for $I_{22}$ and $S_{22}$ on $\phi_{\text {init }}$ can be factored out from the temporal sector, i.e.,

$$
\begin{gathered}
I_{22}\left(\phi_{\text {init }}, t\right) \sim I_{22}^{m}\left(\phi_{\text {init }}\right) T_{I}(t), \\
S_{22}\left(\phi_{\text {init }}, t\right) \sim S_{22}^{m}\left(\phi_{\text {init }}\right) e^{-i \phi_{\text {init }}} T_{S}(t),
\end{gathered}
$$

where $T_{I}(t)$ and $T_{S}(t)$ are two complex functions, corresponding to the temporal evolution of the mass and current quadrupole waves, respectively. Since $T_{I}(t)$ and $T_{S}(t)$ do not depend on $\phi_{\text {init }}$, they represent the common features of all SKd binaries. We will explore the features of $T_{I}(t)$ and $T_{S}(t)$ in Sec. IV. 
$\mathscr{A}^{(I)}$
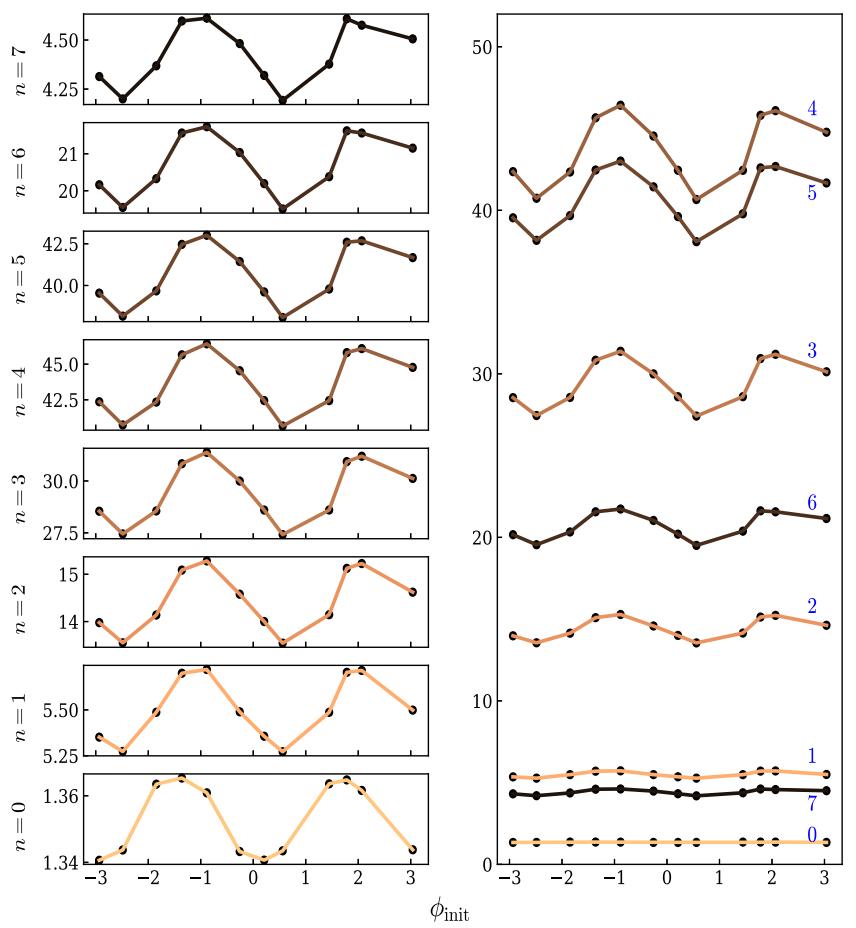

(a) mass quadrupole wave
$\mathscr{A}^{(S)}$
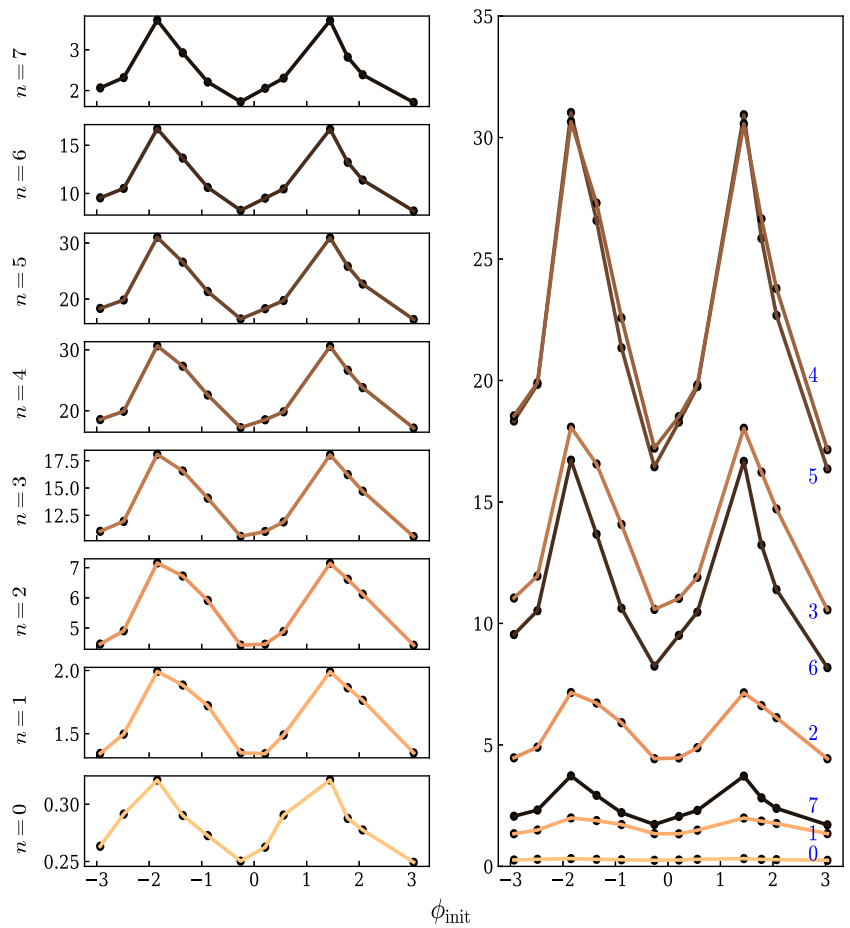

(b) current quadrupole wave

FIG. 2. QNM magnitudes versus $\phi_{\text {init }}$ for mass $\left(\mathcal{A}^{(I)}\right)$ and current $\left(\mathcal{A}^{(S)}\right)$ quadrupole waves. Data are from 12 of our NR simulations listed in Table I. All BBH systems are in the SKd configuration. Fig. 2(a) corresponds to $\mathcal{A}^{(I)}$, where the left eight panels are the zoom-in plot for each overtone. The overtone index $n$ is in descending order. Similarly, Fig. 2(b) corresponds to $\mathcal{A}^{(S)}$. The spectra peak at $n=4$ (because the $n=4$ amplitude is largest), and patterns are roughly periodic with a period $2 \pi$. Examining the zoomed in plots, it can be seen that, approximately, the patterns are the same for all $n$ (up to a scaling factor).

On the other hand, the progenitor configuration, at least $\phi_{\text {init }}$, is encoded mainly in two functions $I_{22}^{m}\left(\phi_{\text {init }}\right)$ and $S_{22}^{m}\left(\phi_{\text {init }}\right)$. Figure 3 exhibits that to the leading order, $\varphi_{n}^{(I)}$ is insensitive to $\phi_{\text {init }}$, while
$\varphi_{n}^{(S)} \propto-\phi_{\text {init }}$. As a result, $I_{22}^{m}\left(\phi_{\text {init }}\right)$ and $S_{22}^{m}\left(\phi_{\text {init }}\right)$ can be regarded approximately as two real functions. Thus the phase difference between $I_{22}$ and $S_{22}, \Delta \Phi_{\text {IS }}$, is roughly linear in $\phi_{\text {init }}$. We will explore Eq. (7) more carefully later
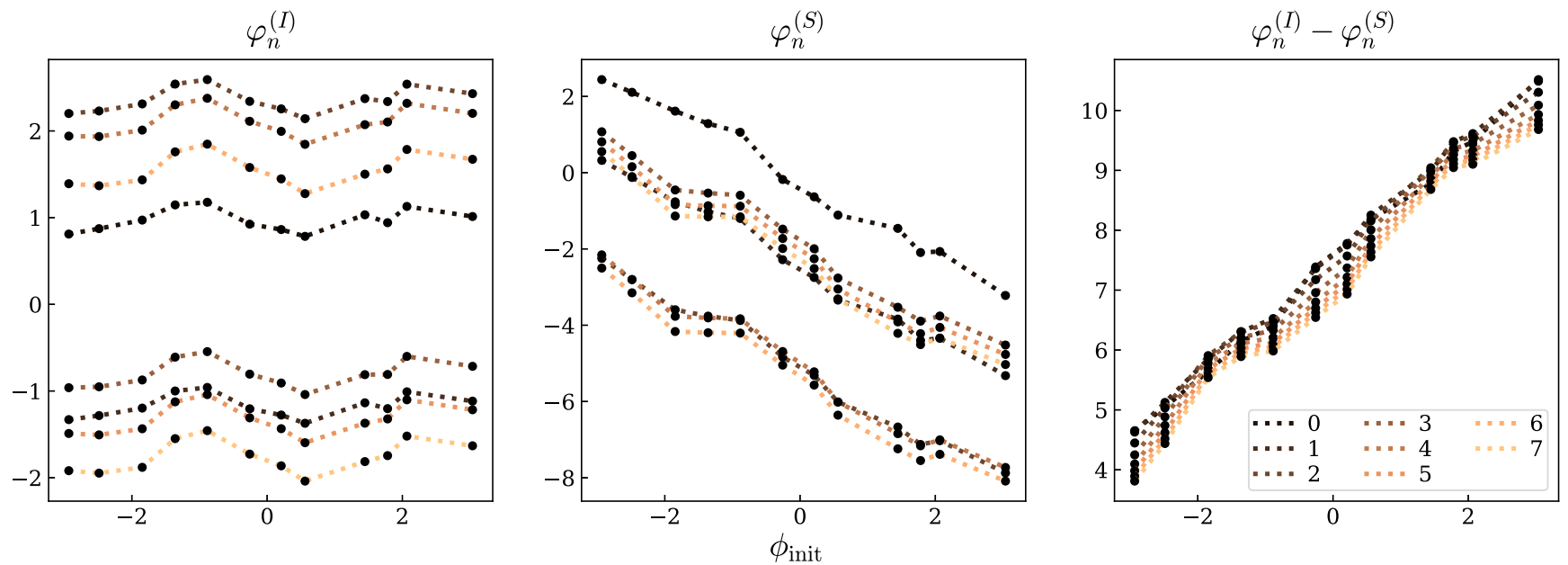

FIG. 3. The dependence of $\varphi_{n}^{(I)}, \varphi_{n}^{(S)}$, as well as their difference, on $\phi_{\text {init. }}$ It turns out that $\varphi_{n}^{(I)}$ is roughly insensitive to $\phi_{\text {init }}$, whereas $\varphi_{n}^{(S)}$ is approximately linear in $\phi_{\text {init }}$. 


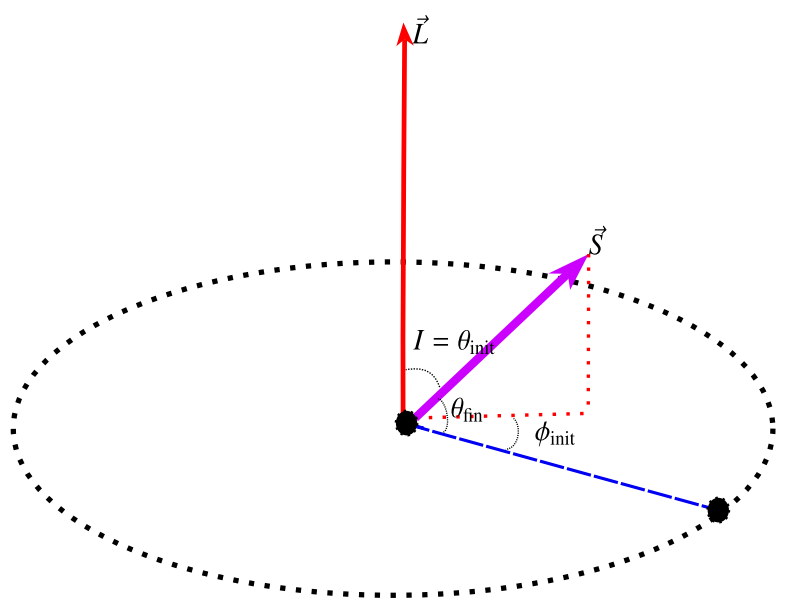

FIG. 4. An illustration for the EMRI-parametrization $\left(I, \theta_{\text {fin }}\right)$ and the SKd-parametrization $\left(\theta_{\text {init }}, \phi_{\text {init }}\right)$. The origin is chosen to be one of the BHs. Following the discussion of Hughes et al. [105-107], $I$ is defined to be the angle between $\boldsymbol{L}$ (the red arrow) and $\boldsymbol{S}$ (the purple arrow), while $\theta_{\text {fin }}$ is the angle between the $\boldsymbol{S}$ (the purple arrow) and the orbital separation vector (the blue dashed line). For the SKd-parametrization, $\phi_{\text {init }}$ is the angle between the in-plane spin (the red dashed horizontal line) and the orbital separation vector (the blue dashed line), whereas $\theta_{\text {init }}$ is the angle between $\boldsymbol{L}$ (the red arrow) and $\boldsymbol{S}$ (the purple arrow). The connection between two parametrizations is given by Eq. (8).

in Sec. III, as well as extending to the full $\left(\chi_{\text {init }}, \theta_{\text {init }}, \phi_{\text {init }}\right)$ parameter space.

\section{Full $\left(\theta_{\text {init }}, \phi_{\text {init }}\right)$ dependence and correspondence with the extreme mass-ratio case}

In the case of EMRI, Hughes et al. [105-107] investigated the ringdown spectra of $h_{2, \pm 2}$ modes rather than $I_{22}$ and $S_{22}$. In order to make a connection to their studies, we now turn our attention to $h_{2, \pm 2}$.

\section{Mapping between SKd and EMRI system parameters}

Hughes et al. [105-107] parametrized EMRIs with two geometric quantities $\theta_{\text {fin }}$ and $I$ (see Fig. 1 of Ref. [105]), where $I \in[0, \pi]$ is the angle between the spin of the primary $\mathrm{BH}$ and the orbital angular momentum, while $\theta_{\text {fin }}$ is the angle between the spin of primary $\mathrm{BH}$ and the orbital separation vector (at the moment of plunge), satisfying $\left|\cos \theta_{\text {fin }}\right| \leq \sin I$. For SKd systems, we can find the counterparts of $\left(I, \theta_{\text {fin }}\right)$ if we treat one of the BHs as the "primary" object. Below we still use the same notation, namely $\left(I, \theta_{\text {fin }}\right)$, to refer to these two angles. As shown in Fig. 4, we pick the primary $\mathrm{BH}$ to be the center of the coordinates. $I$ is still defined to be the angle between $\boldsymbol{L}$ (the red arrow) and $S$ (the purple arrow), while $\theta_{\text {fin }}$ remains to be the angle between the $S$ (the purple arrow) and the orbital separation vector (the blue dashed line). The relations between $\left(I, \theta_{\text {fin }}\right)$ and our parametrization (namely $\theta_{\text {init }}$ and $\phi_{\text {init }}$, see Fig. 1) read

$$
I=\theta_{\text {init }}, \quad \cos \theta_{\text {fin }}=\sin \theta_{\text {init }} \cos \phi_{\text {init }} .
$$

We want to emphasize there are two major differences in the parametrization of EMRIs and SKds. First, the parameters for EMRIs are defined at the moment of plunge, whereas, in our case, it becomes difficult to find well-defined quantities at the merger, thus we use the initial geometry instead (at a reference time during the inspiral stage). Second, for EMRIs, $\pi>I>\pi / 2$ represents the retrograde motion of the small body, and hence the retrograde QNMs dominate in the ringdown signal. By contrast, only the prograde QNMs are excited for SKd systems [see Eq. (4)].

With the purpose of exploring full parameter space of $I$ and $\theta_{\text {fin }}$, we now use the surrogate model NRSur7dq4. Comparing against NR ringdowns, even though NRSur7dq4 has mismatches of order $\sim 3 \times 10^{-4}$, we find that it is not accurate enough to reproduce the correct final mass and spin, in agreement with Ref. [116]. Mismatches of order $10^{-6}$ in the ringdown may be necessary to achieve this. Therefore we fix the values of the final mass and spin to the NR values (coming from NRSur7dq4Remnant) while fitting the mode amplitudes to NRSur7dq4. In addition, we consider only the fundamental mode $(n=0)$.

The results for $\mathcal{A}_{220}$ and $\psi_{220}$ are shown in the first row of Fig. 5. Similar to Refs. [105,107], we use two colors to stand for the sign of $\dot{\theta}_{\text {fin }}$, which was used in the EMRI case to represent the moving direction at the plunge $\left(\dot{\theta}_{\text {fin }}>0\right.$ means that the small particle moves toward the south pole of the Kerr $\mathrm{BH}$, and vice versa). In our case, $\dot{\theta}_{\text {fin }}$ is determined by the sign of $\sin \phi_{\text {init }}$. Comparing to Fig. 3 of Ref. [105], we can see the dependence is similar, although the absolute value of $\mathcal{A}_{220}$ differs.

In the second and third rows of Fig. 5, we present how mode amplitudes depend on $\cos \theta_{\text {fin }}$ for several $I$ slices [Eq. (8)]. Those are direct analogs Fig. 4 of Ref. [107]. It is interesting to note that $\mathcal{A}_{2,+20}$ and $\mathcal{A}_{2,-20}$ are symmetric about the axis of $\cos \theta_{\text {fin }}=0$, so are the patterns for $\psi_{2,+20}$ and $-\psi_{2,-20}$. The other intriguing feature is that the patterns for $I$ and $\pi-I$ are similar.

For overtones $\mathcal{A}_{2,+2 n}(n>0)$, NRSur7dq4 is not accurate enough to provide any prediction, so we use our SKd4 runs instead (see Table I), which corresponds to the $I=\pi / 2$ slice. We translate our previous results in Fig. 2 and 3 to the cases of $h_{2, \pm 2}$ based on Eq. (6). Results are shown in Fig. 6. We can see the patterns for high- $n$ are more distorted.

\section{Understanding the QNM excitation of $h_{2, \pm 2}$ in terms of $\left(I_{22}, S_{22}\right)$}

It turns out that the features in the amplitudes $\mathcal{A}_{2, \pm 2,0}$ that we discussed in Sec. II C 1 can be understood based on what we have learned about $\left(I_{22}, S_{22}\right)$. In order to translate our previous results about $\left(I_{22}, S_{22}\right)$ to $h_{2, \pm 2}$, we use the inverse of Eq. (6) 

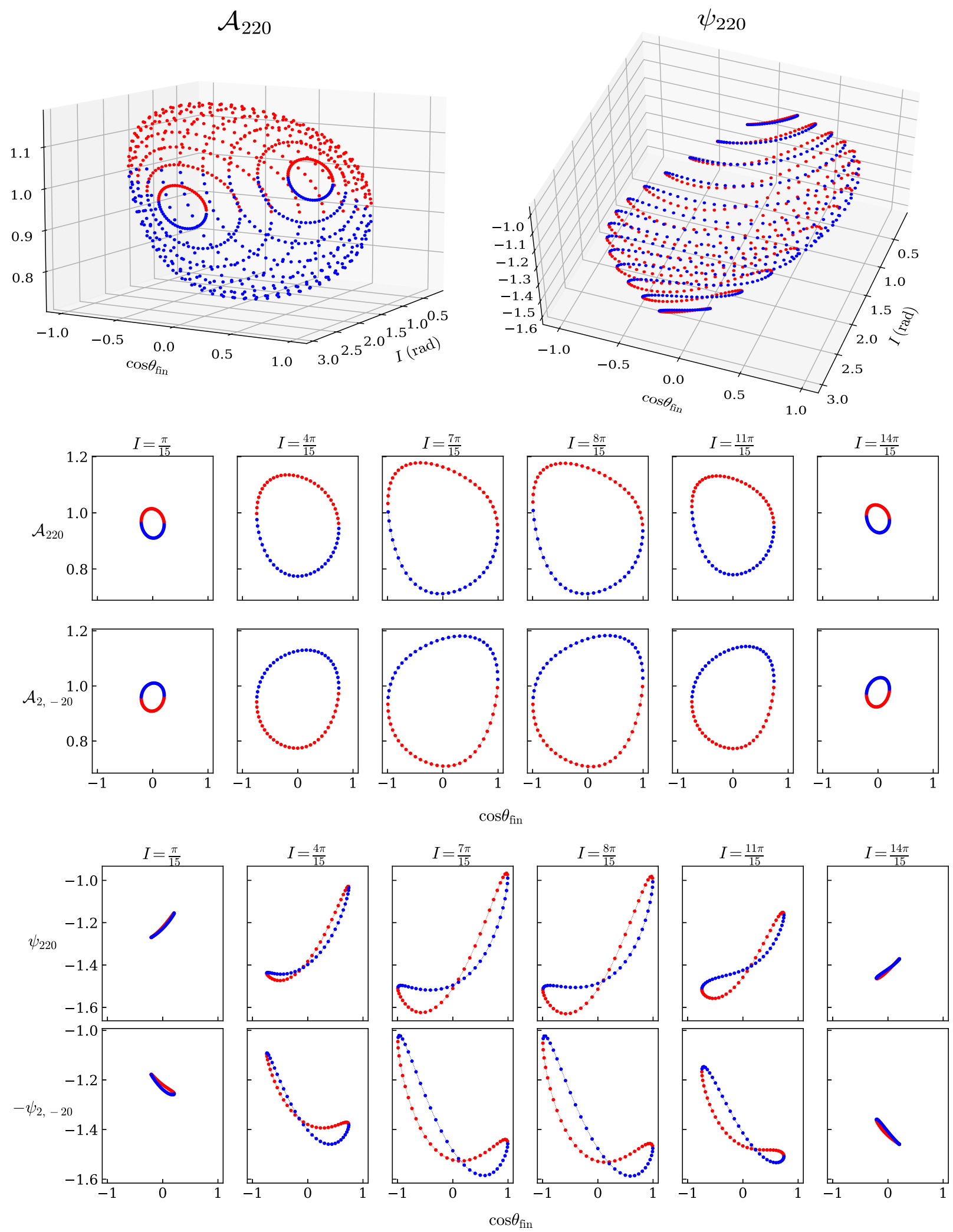

FIG. 5. The fundamental mode amplitude and phase versus initial spin configuration $\left(I, \cos \theta_{\text {fin }}\right)$. Those two independent variables are chosen since they coincide with the variables used in Ref. [105] [see Eq. (8)]. Data are obtained from NRSur7dq4. All BBH systems are in the SKd configuration with $\chi_{\text {init }}=0.4$. Points are drawn with two colors, where blue stands for $\sin \phi_{\text {init }}<0$ while red for sin $\phi_{\text {init }}>0$. The second and fourth rows are results of $h_{2,2}$ for some $I$-slices, while the third and fifth rows correspond to $h_{2,-2}$. 

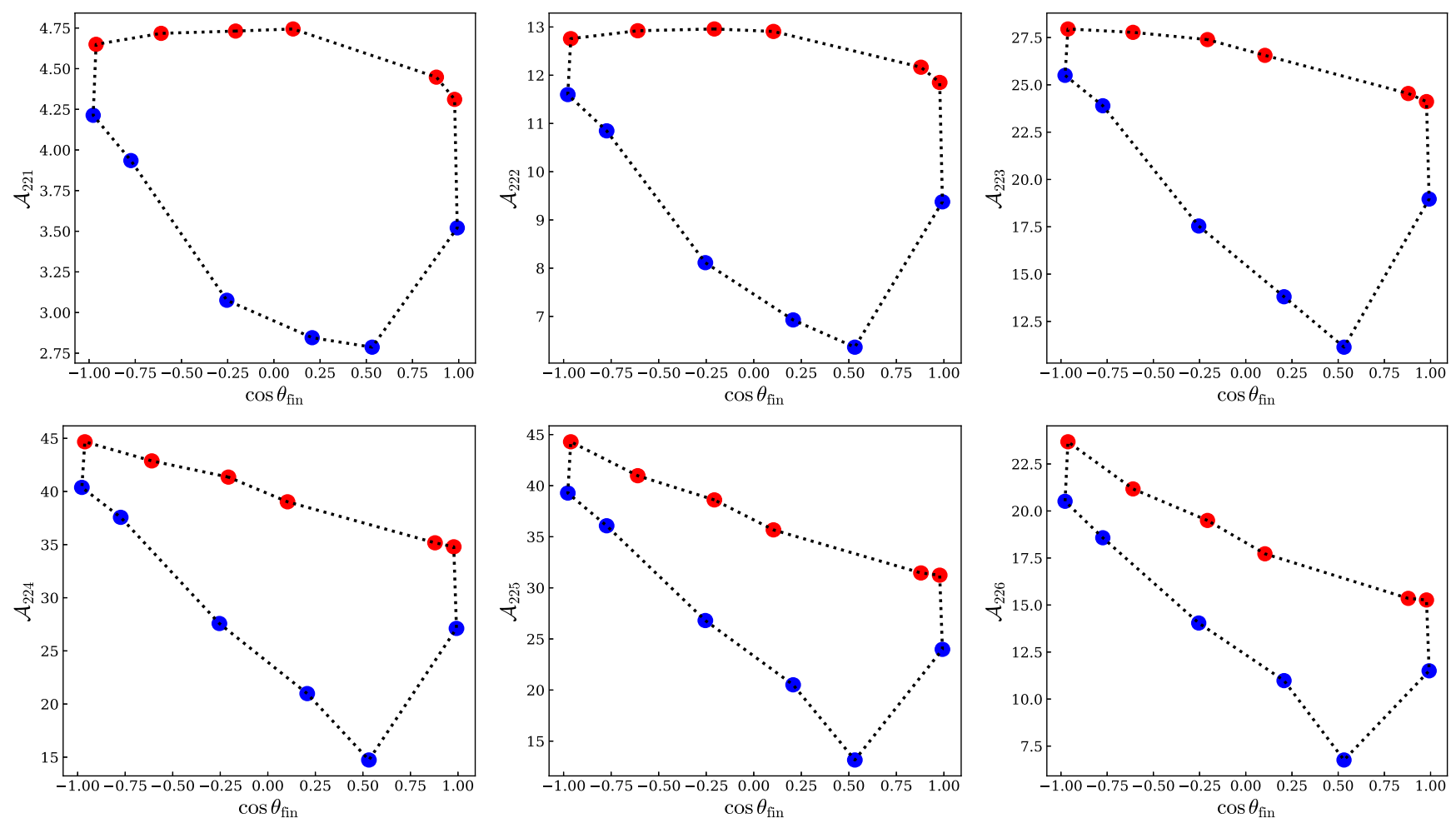

FIG. 6. The overtone mode amplitudes $\mathcal{A}_{2,+2 n}(n=1-6)$ versus $\cos \theta_{\text {fin }}$, with the same convention as Fig. 5. The data are from our SKd4 runs listed in Table I, which corresponds to $I=\pi / 2$.

$$
\mathcal{A}_{2, \pm 2 n} e^{ \pm i \psi_{2, \pm 2 n}}=\frac{1}{\sqrt{2}}\left[\mathcal{A}_{n}^{(I)} e^{i \varphi_{n}^{(I)}} \mp i \mathcal{A}_{n}^{(S)} e^{i \varphi_{n}^{(S)}}\right]
$$

and hence

$$
\mathcal{A}_{2, \pm 2 n}^{2}=\frac{1}{2}\left[\mathcal{A}_{n}^{(I) 2}+\mathcal{A}_{n}^{(S) 2} \mp 2 \mathcal{A}_{n}^{(I)} \mathcal{A}_{n}^{(S)} \sin \left(\varphi_{n}^{(I)}-\varphi_{n}^{(S)}\right)\right]
$$

As we shall explore later in Secs. III D and III E [see Eqs. (33) and (38)], we have two dependencies

$$
\begin{aligned}
& \mathcal{A}_{n}^{(I)}\left(I, \theta_{\text {fin }}\right) \sim \text { const. }+\mathcal{O}\left(v^{4}\right), \\
& \mathcal{A}_{n}^{(S)}\left(I, \theta_{\text {fin }}\right) \sim v^{2} \sin I+\mathcal{O}\left(v^{4}\right),
\end{aligned}
$$

where we have omitted specific numerical coefficients that are independent from $I$ and $\theta_{\text {fin }}$, and $v^{2}$ is a parameter to keep track of the order of approximation (In fact, as we shall show in Sec. III D, $v$ is the orbital velocity that is widely used in the post-Newtonian theory.). Furthermore, we have

$$
\varphi_{n}^{(I)}-\varphi_{n}^{(S)} \sim \phi_{\text {init }}+\text { const. }
$$

Using the above simple dependences of $\left[\mathcal{A}_{n}^{(I)}, \mathcal{A}_{n}^{(S)}, \varphi_{n}^{(I)}-\right.$ $\left.\varphi_{n}^{(S)}\right]$ on $I$ and $\phi_{\text {init }}$, we obtain:
$\mathcal{A}_{2, \pm 2 n} \sim$ const. $\pm v^{2} \sin I \sin \left(\phi_{\text {init }}+\right.$ const. $)+\mathcal{O}\left(v^{4}\right)$.

As a result, for each $I$-slice (i.e., $\theta_{\text {init }}{ }^{-}$slice), the $\mathcal{A}_{2, \pm 20}-$ $\cos \theta_{\text {fin }}$ pattern is an approximate Lissajous-like curve (with identical frequencies), distorted by the higher order term containing $v^{4}$. The variation depends on $I$, which vanishes when $I=0, \pi$, and is maximal when $I=\pi / 2$. Physically speaking, $\mathcal{A}_{2, \pm 2 n}$ depends sensitively on $\phi_{\text {init }}$ when the spins of two BHs lie entirely in the orbital plane (see Fig. 1), but does not change with $\phi_{\text {init }}$ as the spins are (anti)parallel with the orbital angular momentum.

In addition, Eq. (13) implies that $\mathcal{A}_{220}$ and $\mathcal{A}_{2,-20}$ are related by a transformation $\phi_{\text {init }} \rightarrow \phi_{\text {init }}+\pi$, i.e., $\cos \theta_{\text {fin }} \rightarrow$ $-\cos \theta_{\text {fin }}$ [see Eq. (8)]. This transformation represents the interchange of the in-plane spins for two BHs (see Fig. 1). In fact, as we shall study in Sec. III B, this conclusion can be generalized to the entire evolution regime (not only the ringdown phase). The symmetry of the SKd system results in [see Eq. (26)]

$$
\begin{aligned}
& h_{\ell m}\left(\pi-I, \phi_{\text {init }}\right)=(-1)^{m} h_{\ell m}\left(I, \phi_{\text {init }}\right), \\
& h_{\ell m}\left(I, \phi_{\text {init }}+\pi\right)=(-1)^{\ell} h_{\ell,-m}^{*}\left(I, \phi_{\text {init }}\right),
\end{aligned}
$$

i.e., $\mathcal{A}_{2, \pm 2 n}$ remains unchanged when $I \rightarrow \pi-I$ (two BHs interchange their $z$-component spins), and $\mathcal{A}_{2,+2 n} \rightarrow \mathcal{A}_{2,-2 n}$ and $\psi_{2,+20} \rightarrow-\psi_{2,-20}$ when $\phi_{\text {init }} \rightarrow \phi_{\text {init }}+\pi$ (two BHs 
interchange their in-plane spins). ${ }^{1}$ In Fig. 5, we can clearly see the patterns for $\mathcal{A}_{220}$ and $\mathcal{A}_{2,-20}$, as well as the patterns for $\psi_{2,+20}$ and $-\psi_{2,-20}$, are symmetric about the $\cos \theta_{\text {fin }}=0$ axis. Meanwhile, the patterns for $\mathcal{A}_{2 \pm 20}$ are symmetric about the $I=\pi / 2$ axis.

\section{THE FEATURE OF MASS AND CURRENT QUADRUPOLE WAVES}

In the last section, we explored how QNMs are excited with different initial parameters $\left(\theta_{\text {init }}, \phi_{\text {init }}\right)$. We now aim to study features of ringdown more quantitatively. In particular, we focus on the mass $\left(I_{22}\right)$ and current $\left(S_{22}\right)$ quadrupole waves of SKd systems, and relate their features to $\left(\chi_{\text {init }}, \theta_{\text {init }}, \phi_{\text {init }}\right)$. Moreover, since kick velocity is one of the important quantities that reflects SKd systems' properties, we also include it in our study.

\section{A. A brief review}

This subsection briefly reviews some facts about the gravitational recoil. In particular, we relate the kick velocity to the radiative mass and current quadrupole waves.

It has been shown that for a SKd system, the kick magnitude can be estimated with a simple formula [78-80]

$$
v_{f} \sim \chi_{\text {init }} \sin \left(\phi_{\text {init }}-\phi_{\text {init }}^{(0)}\right) \sin \theta_{\text {init }},
$$

where $\phi_{\text {init }}^{(0)}$ is a constant. Equation (14) is based on the computation of linear momentum carried away by GW [117]

$$
\dot{P}_{z}=\lim _{D \rightarrow \infty} \frac{1}{24 \pi}\left(\dot{h}_{22} \dot{h}_{22}^{*}-\dot{h}_{2,-2} \dot{h}_{2,-2}^{*}\right),
$$

where $*$ stands for complex conjugate, $D$ is the distance between the source and the observer, and the $z$-axis is in the direction of orbital angular momentum. Here we have ignored the effects of other modes since they are negligible.

In terms of $I_{22}$ and $S_{22}$ [Eq. (3)], Eq. (15) can also be written as

$$
\dot{P}_{z}=-\frac{1}{12 \pi} \operatorname{Im} \dot{I}_{22} \dot{S}_{22}^{*}
$$

and the final kick velocity is given by

$$
\begin{aligned}
m_{f} v_{f} & =\frac{1}{12 \pi} \operatorname{Im} \int \dot{I}_{22} \dot{S}_{22}^{*} d t \\
& =\frac{1}{12 \pi} \operatorname{Im} \int\left|\dot{I}_{22}\right|\left|\dot{S}_{22}\right| e^{i \Phi_{i s}} d t
\end{aligned}
$$

with $\Phi_{i \dot{S}}$ the phase difference between $\dot{I}_{22}$ and $\dot{S}_{22}$. Note that the change of sign from Eq. (16) to (17) is a result of linear momentum conservation. In Fig. 7, we show the time

\footnotetext{
${ }^{1}$ Equivalently, $\cos \theta_{\text {fin }} \rightarrow-\cos \theta_{\text {fin }}$.
}

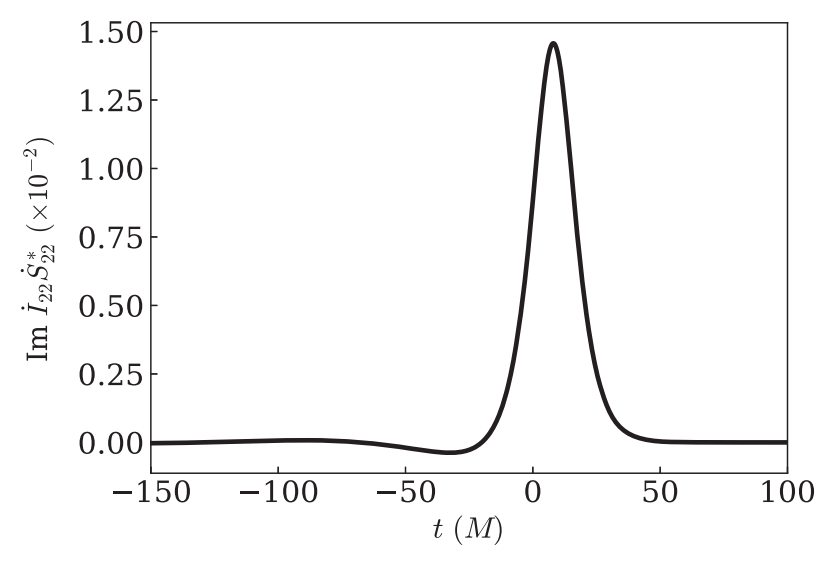

FIG. 7. The integrand of Eq. (17) for the SKd4-"06" system. The majority kick velocity is accumulated around $t \sim 0 M$, and the final kick is $4.75 \times 10^{-3}$.

evolution of the above-mentioned integrand for SKd4-"06" (cf. Table I). We can see that most of the kick velocity is accumulated around $t \sim 0 M$.

During the inspiral stage, it was shown that $I_{22}$ and $S_{22}$ are related to the source quadrupole moments. ${ }^{2}$ At the leading order, from Refs. [70,118], we write

$$
\begin{gathered}
I_{22}(t)=-\frac{M}{2} \sqrt{\frac{2 \pi}{5}} \frac{d^{2}}{d t^{2}} r(t)^{2} e^{-2 i \phi(t)}, \\
S_{22}(t)=\sqrt{\frac{2 \pi}{5}} \chi \sin \theta(t) \frac{d^{2}}{d t^{2}} r(t) e^{-i \phi(t)-i \phi_{\mathrm{pre}}(t),}
\end{gathered}
$$

where $M$ is the total mass of the BBH system; $\chi$ is the dimensionless spin of an individual $\mathrm{BH} ; \phi(t)$ and $r(t)$ are the orbital phase and separation, respectively; $\theta(t)$ is the polar angle of the spin; and $\phi_{\text {pre }}(t)$ is the precession angle (the azimuthal angle of the in-plane spin component). Note that at the initial time $t_{\text {init }}$

$$
\theta_{\text {init }}:=\theta\left(t_{\text {init }}\right), \quad \phi_{\text {init }}:=\phi\left(t_{\text {init }}\right) .
$$

For instance, we choose SKd4-"03" (see Table I) and compare its radiative multipolar waves $I_{22}$ and $S_{22}$ to PN formulas in Eq. (18). We read off the values of $r(t), \theta(t)$, and $\phi(t)$ directly from the outputs of NR simulation. The results are shown in Fig. 8. For comparison, we also fit the ringdown signal with QNMs (7 overtones), starting from $t=0 M$. We can see the Newtonian formulas can accurately model the phase evolution up to $t \sim-250 M$. Meanwhile, both $I_{22}$ and $S_{22}$ are described by 7 overtones accurately from $t=0 M$.

\footnotetext{
${ }^{2}$ Hereafter we shall not distinguish the source quadrupole moment and the (radiative) quadrupole wave since it will not cause any confusion.
} 

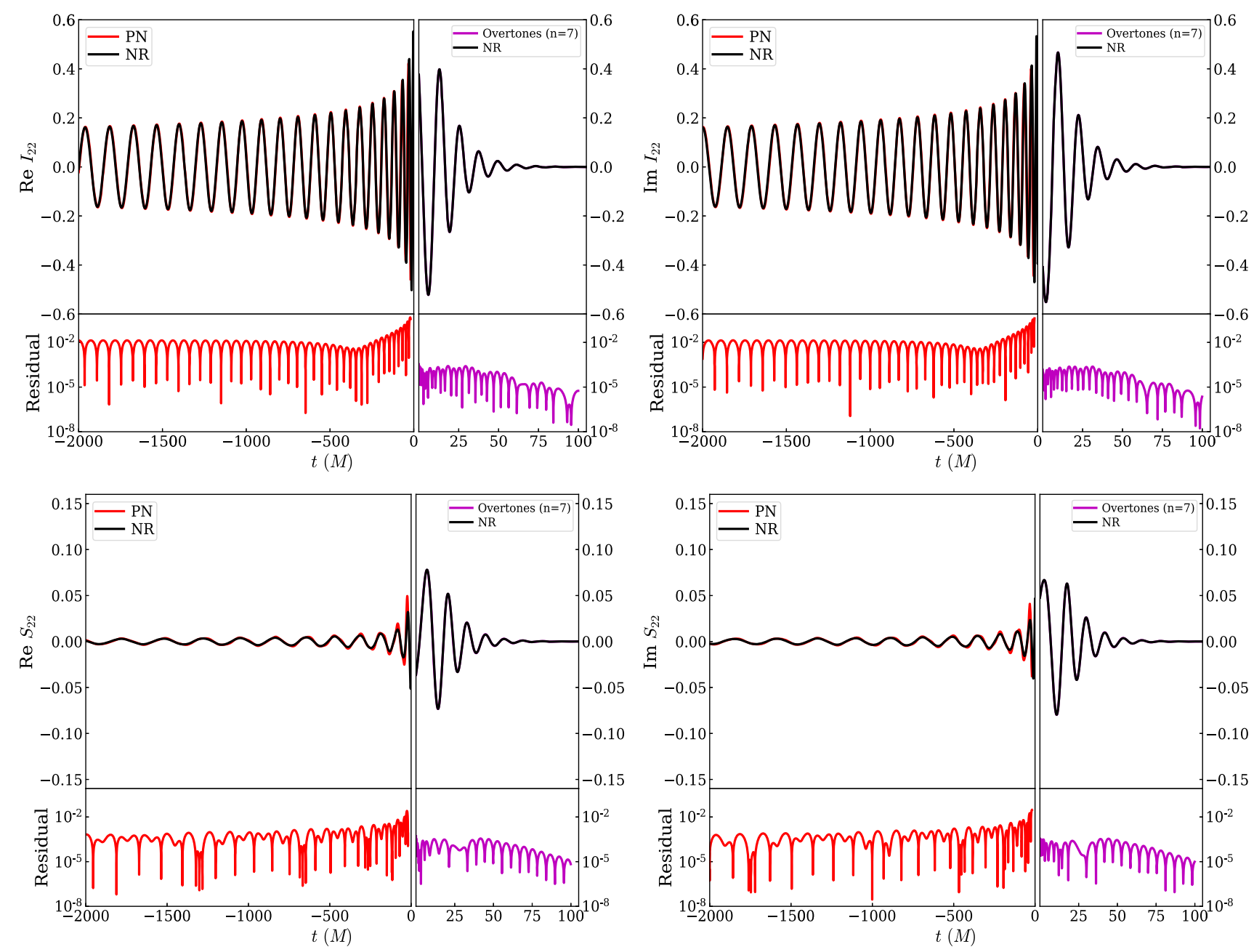

FIG. 8. The time evolution of $I_{22}$ (upper row) and $S_{22}$ (bottom row) for the SKd4-"03" system, where $t=0 M$ stands for the peak of strain amplitude. The mass $\left(I_{22}\right)$ and current $\left(S_{22}\right)$ quadrupole waves are compared to PN formulas [Eq. (18)] during the inspiral stage, and to QNMs (7 overtones) in the ringdown regime.

In the rest of this section, we shall discuss how $I_{22}$ and $S_{22}$ depend on $\left(\chi_{\text {init }}, \theta_{\text {init }}, \phi_{\text {init }}\right)$, and apply our understanding to the gravitational recoil.

\section{B. Symmetry properties of $\boldsymbol{I}_{22}$ and $S_{22}$}

Before exploring the detailed relations between $\left(I_{22}, S_{22}\right)$ and $\left(\chi_{\text {init }}, \theta_{\text {init }}, \phi_{\text {init }}\right)$, we first take advantage of the symmetry of SKd systems, and study its implication on $\left(I_{22}, S_{22}\right)$. As shown in Fig. 9, there are three SKd binaries, where (a) and (b) are related by a parity transformation, i.e., two BHs interchange their locations while having their spin directions fixed, recalling that spin is an axial vector, which is not changed by the parity transformation. On the other hand, we rotate the whole system in (b) about the orbital angular momentum by $\pi$, and obtain (c). We use wavy lines to stand for the GW propagating direction, and $(l, \beta)$ are the coordinates of the observer in (b), as defined in Eq. (2). The coordinates of observers in (a) and (c) are transformed accordingly. As discussed in Eq. (2), $h(t, l, \beta)$ can be decomposed into the extrinsic part ${ }_{-2} Y_{\ell m}(l, \beta)$ and the intrinsic part $h_{\ell m}\left(\theta_{\text {init }}, \phi_{\text {init }}\right){ }^{3}$. Here we omit $\chi_{\text {init }}$ in the argument of $h_{\ell m}$ since it has no impact on the transformation in question.

Figures 9(a) and 9(b) are related by a parity transformation; hence, we have (see the Appendix C for more details)

$$
h^{(a)}=h^{(b) *},
$$

i.e.,

$$
\begin{aligned}
& h_{\ell m}\left(\theta_{\text {init }}, \phi_{\text {init }}\right)_{-2} Y_{\ell m}(l, \beta) \\
& \quad=h_{\ell m}^{*}\left(\pi-\theta_{\text {init }}, \phi_{\text {init }}+\pi\right)_{-2} Y_{\ell m}{ }^{*}(\pi-\imath, \pi+\beta) .
\end{aligned}
$$

\footnotetext{
${ }^{3}$ We use $\left(\theta_{\text {init }}, \phi_{\text {init }}\right)$ to stand for the spin of $\mathrm{BH}$ on the left. The other spin is determined uniquely by the SKd condition.
} 


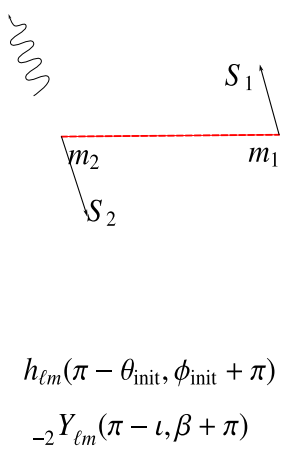

(a)

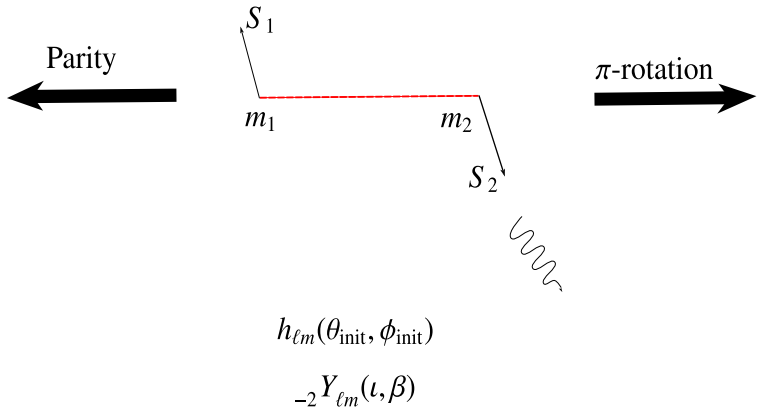

(b)

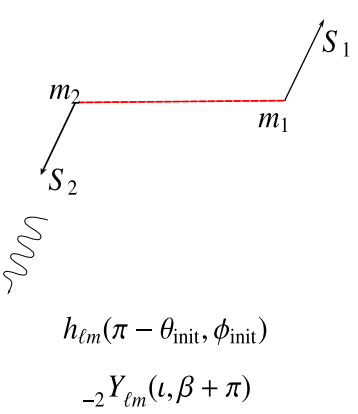

(c)

FIG. 9. Parity inversion of a SKd BBH system. We use arrows to represent the spin direction of BHs, and wavy lines to stand for the GW propagating direction. The complex strain of a SKd system is totally determined by two extrinsic parameters $(l, \beta)$, and three intrinsic parameters $\left(\chi_{\text {init }}, \theta_{\text {init }}, \phi_{\text {init }}\right)$. Here the intrinsic parameters are the spin of the left $\mathrm{BH}$. The properties of the right $\mathrm{BH}$ are determined based on the SKd condition. Figs. 9(a) and 9(b) are related by a parity inversion: two BHs exchange their locations while having their own spins fixed. As a result, the GW propagation direction and both spins change sign, i.e., $(l, \beta) \leftrightarrow(\pi-l, \pi+\beta)$ and $\left(\theta_{\text {init }}, \phi_{\text {init }}\right) \leftrightarrow\left(\pi-\theta_{\text {init }}, \pi+\phi_{\text {init }}\right)$. Figs. 9(b) and 9(c) are related by a $\pi$-rotation about the orbital angular momentum. Thus we have $(\iota, \beta) \leftrightarrow(\iota, \pi+\beta)$ and $\left(\theta_{\text {init }}, \phi_{\text {init }}\right) \leftrightarrow\left(\pi-\theta_{\text {init }}, \phi_{\text {init }}\right)$.

Using the fact that

$$
{ }_{-2} Y_{\ell m}{ }^{*}(\pi-\imath, \pi+\beta)=(-1)^{\ell+m}{ }_{-2} Y_{\ell m}(l, \beta),
$$

we obtain

$h_{\ell m}\left(\theta_{\text {init }}, \phi_{\text {init }}\right)=(-1)^{\ell+m} h_{\ell,-m}^{*}\left(\pi-\theta_{\text {init }}, \phi_{\text {init }}+\pi\right)$.

On the other hand, Figs. 9(b) and 9(c) are related by a global rotation. Therefore, the observable $h(t, l, \beta)$ should not be affected

$$
h^{(b)}=h^{(c)},
$$

i.e.,

$$
\begin{aligned}
& h_{\ell m}\left(\theta_{\text {init }}, \phi_{\text {init }}\right)_{-2} Y_{\ell m}(l, \beta) \\
& \quad=h_{\ell m}\left(\pi-\theta_{\text {init }}, \phi_{\text {init }}\right)_{-2} Y_{\ell m}(l, \pi+\beta) .
\end{aligned}
$$

Recalling that

$$
{ }_{-2} Y_{\ell m}(l, \pi+\beta)=(-1)^{m}{ }_{-2} Y_{\ell m}(l, \beta),
$$

we then have

$$
\begin{gathered}
h_{\ell m}\left(\pi-\theta_{\text {init }}, \phi_{\text {init }}\right)=(-1)^{m} h_{\ell m}\left(\theta_{\text {init }}, \phi_{\text {init }}\right), \\
h_{\ell m}\left(\theta_{\text {init }}, \phi_{\text {init }}+\pi\right)=(-1)^{\ell} h_{\ell,-m}^{*}\left(\theta_{\text {init }}, \phi_{\text {init }}\right),
\end{gathered}
$$

where the first line is the result of Eqs. (24) and (25), and the second line comes from the combination of Eqs. (22) and (26a). Equations (26) give the transformation of $h_{\ell m}$ under $\theta_{\text {init }} \rightarrow \pi-\theta_{\text {init }}$ (two BHs interchange their $z$-component spins) and $\phi_{\text {init }} \rightarrow \phi_{\text {init }}+\pi$ (two BHs interchange their in-plane spins). As we discussed in Sec. II C, Eqs. (26) directly lead to several features revealed in Fig. 5: the patterns for $\mathcal{A}_{2 \pm 20}$, as well as $\psi_{2,+20}$ and $-\psi_{2,-20}$, have a reflective symmetry about the $\cos \theta_{\text {fin }}=0$ axis; and the patterns for $\mathcal{A}_{2 \pm 20}$ are symmetric about the $I=\pi / 2$ axis.

We then apply Eqs. (26) to the case of $I_{\ell m}$ and $S_{\ell m}$, [see Eqs. (3)]

$$
\begin{gathered}
I_{\ell m}\left(\pi-\theta_{\text {init }}, \phi_{\text {init }}\right)=(-1)^{m} I_{\ell m}\left(\theta_{\text {init }}, \phi_{\text {init }}\right), \\
S_{\ell m}\left(\pi-\theta_{\text {init }}, \phi_{\text {init }}\right)=(-1)^{m} S_{\ell m}\left(\theta_{\text {init }}, \phi_{\text {init }}\right), \\
I_{\ell m}\left(\theta_{\text {init }}, \phi_{\text {init }}+\pi\right)=(-1)^{\ell+m} I_{\ell m}\left(\theta_{\text {init }}, \phi_{\text {init }}\right),
\end{gathered}
$$

$S_{\ell m}\left(\theta_{\text {init }}, \phi_{\text {init }}+\pi\right)=(-1)^{\ell+m+1} S_{\ell m}\left(\theta_{\text {init }}, \phi_{\text {init }}\right)$.

One can find the counterpart of Eqs. (27) for EMRIs in Eq. (4.6) of Ref. [107]. Those relations imply that the dependence of $I_{22}$ and $S_{22}$ on $\theta_{\text {init }}$ is symmetric about $\theta_{\text {init }}=\pi / 2$ axis, whereas the dependence of $\left|I_{22}\right|$ and $\left|S_{22}\right|$ on $\phi_{\text {init }}$ have a period $\pi .{ }^{4}$ We shall see these features shortly from numerical results.

\section{Time dependence of $\boldsymbol{I}_{22}$ and $S_{22}$}

After the study of $\left(I_{22}, S_{22}\right)-\left(\chi_{\text {init }}, \theta_{\text {init }}, \phi_{\text {init }}\right)$ dependence enforced by the symmetry, we are in a position to carry out more detailed analyses. Based on the discussion around Eq. (7), for the postmerger evolution of $I_{22}$ and $S_{22}$, their $\phi_{\text {init }}$ dependence can be factored out. In particular, the spin sector of $I_{22}$ is described by a function $I_{22}^{m}\left(\phi_{\text {init }}\right)$, and that of $S_{22}$ is given by $S_{22}^{m}\left(\phi_{\text {init }}\right) e^{-i \phi_{\text {init. }}}$. In fact, those features are

\footnotetext{
${ }^{4}$ Here we use the absolute value for future convenience.
} 

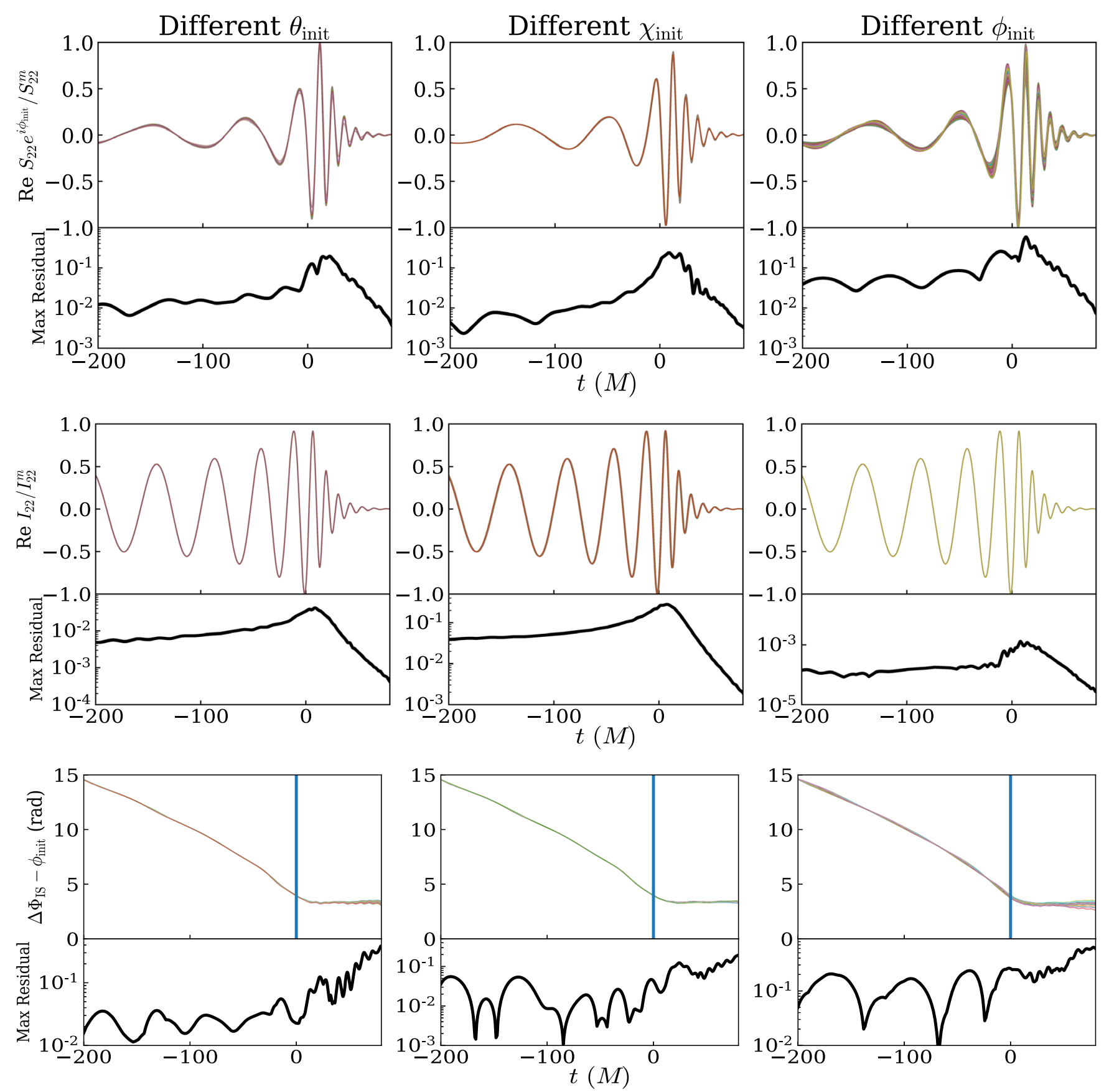

FIG. 10. The time evolution of the real part of the normalized $S_{22}$ (upper row), the real part of the normalized $I_{22}$ (middle row), as well as $\Delta \Phi_{\text {IS }}-\phi_{\text {init }}$ (bottom row), using the SKd BBH configuration from NRSur7dq4. The imaginary part is similar. We sample in total 180 cases with different $\theta_{\text {init }} \in[0, \pi]$ (left column), $\chi_{\text {init }} \in[0,0.8]$ (middle column), and $\phi_{\text {init }} \in[0,2 \pi]$ (right column), and plot them on top of each other. "Max Residual" is defined to be the maximum difference of all cases at each time step. The normalized $I_{22}$ and $S_{22}$ are insensitive to $\left(\chi_{\text {init }}, \theta_{\text {init }}, \phi_{\text {init }}\right)$, to the level of $\sim 0.1 \%-30 \%$.

also consistent with PN predictions, as shown in Eqs. (18): To the leading PN order, $I_{22}$ is independent of $\left(\chi_{\text {init }}, \theta_{\text {init }}, \phi_{\text {init }}\right)$, whereas $S_{22} \sim \chi_{\text {init }} \sin \theta_{\text {init }} e^{-i \phi_{\text {init }} \text {. In light }}$ of the facts, it is reasonable to conjecture that the separability between the spin sector (including $\chi_{\text {init }}, \theta_{\text {init }}, \phi_{\text {init }}$ ) and the temporal sector is preserved throughout the entire process, i.e.,

$$
\begin{gathered}
I_{22}\left(t, \chi_{\text {init }}, \theta_{\text {init }}, \phi_{\text {init }}\right)=I_{22}^{m}\left(\chi_{\text {init }}, \theta_{\text {init }}, \phi_{\text {init }}\right) T_{I}(t), \\
S_{22}\left(t, \chi_{\text {init }}, \theta_{\text {init }}, \phi_{\text {init }}\right)=S_{22}^{m}\left(\chi_{\text {init }}, \theta_{\text {init }}, \phi_{\text {init }}\right) e^{-i \phi_{\text {init }}} T_{S}(t),
\end{gathered}
$$

where $T_{I}(t)$ and $T_{S}(t)$ are two complex functions of time, which are normalized such that they each is equal to 1 at 
the moment when its magnitude is at maximum. As a result, $I_{22}^{m}$ and $S_{22}^{m}$ are in fact the peak values of $I_{22}$ and $S_{22}$, respectively, i.e.,

$$
\begin{aligned}
& I_{22}^{m}\left(\chi_{\text {init }}, \theta_{\text {init }}, \phi_{\text {init }}\right)=\max _{t}\left|I_{22}\left(t, \chi_{\text {init }}, \theta_{\text {init }}, \phi_{\text {init }}\right)\right|, \\
& S_{22}^{m}\left(\chi_{\text {init }}, \theta_{\text {init }}, \phi_{\text {init }}\right)=\max _{t}\left|S_{22}\left(t, \chi_{\text {init }}, \theta_{\text {init }}, \phi_{\text {init }}\right)\right| .
\end{aligned}
$$

We want to emphasize that Eq. (29) is an approximation based on the observation we made in Fig. 3, namely to the leading order $\varphi_{n}^{(I)}$ is insensitive to $\phi_{\text {init }}$, while $\varphi_{n}^{(S)} \propto-\phi_{\text {init }}$. This fact allows us to treat $I_{22}^{m}$ and $S_{22}^{m}$ as two real functions [see the context below Eq. (7)]. The higher order corrections will lead to additional phase factors for both $I_{22}^{m}$ and $S_{22}^{m}$. This is beyond the scope of this work.

To test the accuracy of Eqs. (28) and (29), we use NRSur7dq4 to obtain $I_{22}$ and $S_{22}$ with different initial spin configurations. They are normalized by $I_{22}^{m}$ and $S_{22}^{m} e^{-i \phi_{\text {init }} \text {, }}$ respectively. The results are shown in the first two rows of Fig. 10. To avoid redundancy, we present only the real part since the imaginary part is similar. As we can see, the normalized $I_{22}$ with different $\left(\chi_{\text {init }}, \theta_{\text {init }}, \phi_{\text {init }}\right)$ evolves in a similar way, so does the normalized $S_{22}$. The residuals imply that Eqs. (28) are accurate to $\sim 0.1 \%-$ $30 \%$ throughout the entire evolution. We remark that the accuracy is limited by the approximation adopted in Eq. (29), where $I_{22}^{m}$ and $S_{22}^{m}$ are treated as two real functions and their phases (higher order effects) are not included. If we omit these additional phase terms that are functions of $\left(\chi_{\text {init }}, \theta_{\text {init }}, \phi_{\text {init }}\right)$, there will be a non-negligible increase in the residual. In fact, if we consider only the absolute value of the normalized $I_{22}$ and $S_{22}$, the residual can be decreased by a factor of $1.6 \sim 100$.

Nevertheless, the progenitor's information is primarily described by the peak value of mass and current quadrupole waves, $I_{22}^{m}$ and $S_{22}^{m}$. On the other hand, the temporal evolution, $T_{I}(t)$ and $T_{S}(t)$, encode the common feature of SKd systems. In particular, as we discussed in Sec. III A, the phase difference between $T_{I}(t)$ and $T_{S}(t) e^{-i \phi_{\text {init }} \text {, }}$ denoted by $\Delta \Phi_{\text {IS }}$

$$
\begin{aligned}
\Delta \Phi_{\mathrm{IS}} & \equiv \arg \left(T_{I}\right)-\arg \left(T_{S} e^{-i \phi_{\text {init }}}\right) \\
& \equiv \arg \left(I_{22}\right)-\arg \left(S_{22}\right),
\end{aligned}
$$

is closely related to the gravitational recoil.

We have introduced three quantities, $I_{22}^{m}, S_{22}^{m}$ and $\Delta \Phi_{\mathrm{IS}}$, which are important characteristics of SKd systems. In the rest of this section, we aim to study $I_{22}^{m}, S_{22}^{m}$, and $\Delta \Phi_{\text {IS }}$ more carefully and more quantitatively. In particular, we will show that $I_{22}^{m}$ and $S_{22}^{m}$ are subject to the periodic condition in both $\theta_{\text {init }}-$ and $\phi_{\text {init }}-$ directions, as enforced by the symmetry in Eqs. (27).

\section{The peak of mass quadrupole wave $I_{22}^{m}$}

We saw that $I_{22}^{m}$ is an important characteristic quantity for SKd systems. In fact, it was shown that the remnant BH spin is already encoded in the peak amplitude of the gravitational wave strain [56]. Therefore, it is instructive to study how $I_{22}^{m}$ depends on $\left(\chi_{\text {init }}, \theta_{\text {init }}, \phi_{\text {init }}\right)$.

We first look at our SKd4 NR runs listed in Table I. Figure 11 shows $I_{22}^{m}$ as a function of $\phi_{\text {init }}$. We can see that $I_{22}^{m}$ does depend weakly on $\phi_{\text {init }}$ for all three numerical resolutions, which verifies that the dependence is not a numerical artifact. For comparison purposes, we also show the prediction of NRSur7dq4 with the same BBH system but varying $\phi_{\text {ini }}$. Two results are close. With different $\phi_{\text {init }}$, $I_{22}^{m}$ varies on the level of $\sim 0.36 \%$, around a base value $\sim 0.557$. As discussed earlier, $I_{22}^{m}-\phi_{\text {init }}$ relation is expected to have a period of $\pi$ [Eq. (27)]. However, the black curve is slightly asymmetric. We attribute this to the numerical error of NRSur7dq4. Furthermore, the change of $I_{22}^{m}$ is much smaller than the base value, which is qualitatively consistent with PN predictions, because the variation caused by spin is 2PN [118] smaller than the leading contribution from the orbital mass quadrupole moment [Eq. (18)].

To explore a larger parameter space, we use NRSur7dq4 and plot $I_{22}^{m}$ as a function of $\left(\theta_{\text {init }}, \phi_{\text {init }}\right)$ in Fig. 12, with $\chi_{\text {init }}=0.8$. The pattern exhibits quadrupolar structure, i.e., symmetric about $\theta_{\text {init }}=\pi / 2$, and has a period $\pi$ in the $\phi_{\text {init }^{-}}$ direction. This is consistent with what we obtained in Eq. (27).

To have a better understanding of $I_{22}^{m}$, we use PN prediction of mass quadrupole wave during the inspiral stage [118]

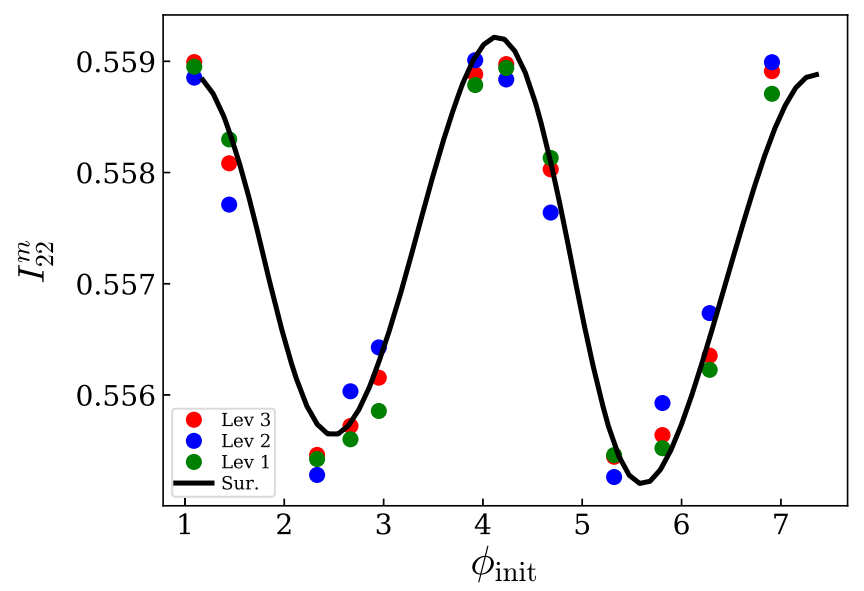

FIG. 11. The peak value of mass quadrupole wave $I_{22}^{m}$ as a function of $\phi_{\text {init }}$. We use SKd4 systems listed in Table I. The black curve is from NRSur7dq4, whereas points are from NR simulations. Colors (labeled by Lev) correspond to numerical resolutions, where "Lev 1" stands for the lowest resolution. Predictions of NRSur7dq4 are consistent with NR results: $I_{22}^{m}$ oscillates with $\phi_{\text {init }}$ on the level of $\sim 0.36 \%$, around a base value $\sim 0.557$. 


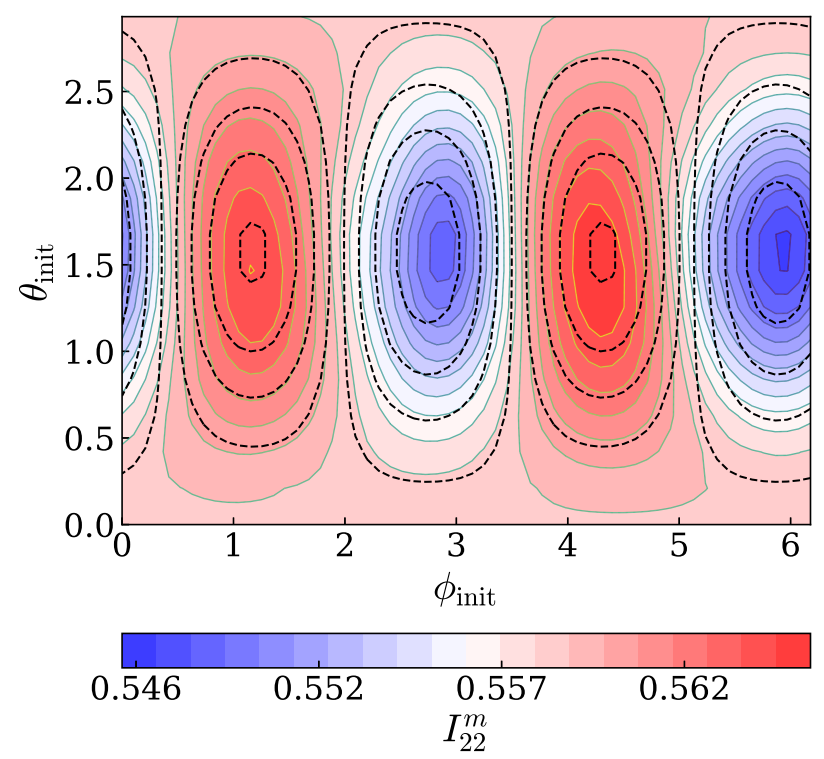

FIG. 12. The peak value of mass quadrupole wave $I_{22}^{m}$ as a function of $\left(\theta_{\text {init }}, \phi_{\text {init }}\right)$, with $\chi_{\text {init }}=0.8$ (SKd configuration). Results are from NRSur7dq4. The pattern is symmetric about $\theta_{\text {init }}=\pi / 2$, and has a period $\pi$ in the $\phi_{\text {init }}$-direction, consistent with Eq. (27). The contours with dashed lines are the prediction of PN-inspired counterpart in Eq. (33).

$$
\mathcal{I}_{22}=\mathcal{I}_{\text {orb }}+\mathcal{I}_{S 1}+\mathcal{I}_{S 2}
$$

where

$$
\begin{gathered}
\mathcal{I}_{\text {orb }}=-\frac{M}{2} \sqrt{\frac{2 \pi}{5}} r^{2} e^{i \phi} \\
\mathcal{I}_{S 1}=\sqrt{\frac{2 \pi}{5}} \frac{M^{3}}{16} \chi_{\text {init }}^{2} \sin ^{2} \theta_{\text {init }} e^{-2 i \phi_{\text {init }}}, \\
\mathcal{I}_{S 2}=-\sqrt{\frac{2 \pi}{5}} \frac{M^{3}}{16} \chi_{\text {init }}^{2} v^{2}
\end{gathered}
$$

with $v \sim \sqrt{M / r}$ the velocity of an individual BH. In Eq. (31), the leading contribution from the orbital sector $\mathcal{I}_{\text {orb }}$ is modified by the spin sector $\mathcal{I}_{S 1,2}$. As discussed in Refs. $[119,120]$, the amplitudes of ringdown waveforms in different $(\ell, m)$ modes are related to those of the corresponding modes during the inspiral stage. Therefore, we can write down a fitting formula for the relation $I_{22}^{m}-\left(\chi_{\text {init }}, \theta_{\text {init }}, \phi_{\text {init }}\right)$, inspired by Eq. (31) and the definition of $I_{22}$ in Eq. (3),

$$
\begin{aligned}
I_{22}^{m}= & Q_{\text {orb }}+Q_{S 1} \chi_{\text {init }}^{2} \sin ^{2} \theta_{\text {init }} \sin 2\left(\phi_{\text {init }}+\phi_{0}\right) \\
& +Q_{S 2} \chi_{\text {init }}^{2},
\end{aligned}
$$

where $Q_{\text {orb }}, Q_{S 1}, Q_{S 2}$ are constants. Their fitted values are listed in Table II, and the contours of Eq. (33) are plotted as dashed lines in Fig. 12. We note that Eq. (33) was
TABLE II. The coefficients in Eq. (33) by fitting to the NRSur7dq4 data. The values of $Q_{\text {orb }} / Q_{S 1}$ and $Q_{S 2} / Q_{S 1}$ are close to the PN predictions in Eqs. (34).

\begin{tabular}{lccccc}
\hline \hline$Q_{\text {orb }}$ & $Q_{S 2}$ & $Q_{S 1}$ & $\tan 2 \phi_{0}$ & $Q_{\text {orb }} / Q_{S 1}$ & $Q_{S 2} / Q_{S 1}$ \\
\hline 0.557 & $2.72 \times 10^{-3}$ & $12.2 \times 10^{-3}$ & -0.98 & 45.7 & 0.22 \\
\hline \hline
\end{tabular}

applied to understand the features of $\mathcal{A}_{n}^{(I)}$ in Sec. II C [see Eq. (11)], where we used the fact that $\mathcal{A}_{n}^{(I)}$ is insensitive to the overtone index $n$ and we ignored the mixing between overtones.

Three terms in Eq. (33) correspond to $\mathcal{I}_{\text {orb }}, \mathcal{I}_{S 1}$, and $\mathcal{I}_{S 2}$, respectively. They imply that

$$
\begin{gathered}
Q_{\text {orb }} / Q_{S 1} \sim\left|\mathcal{I}_{\text {orb }}\right| \chi_{\text {init }}^{2} /\left|\mathcal{I}_{S 1}\right| \sim 8 \frac{r^{2}}{M^{2}} \sim 72, \\
Q_{S 2} / Q_{S 1} \sim\left|\mathcal{I}_{S 2}^{i j}\right| \sin ^{2} \theta_{\text {init }} /\left|\mathcal{I}_{S 1}^{i j}\right| \sim v^{2} \sim 0.3,
\end{gathered}
$$

where the formula is evaluated at $r=3 M$, i.e., the radius of the light ring. In fact, values in Eq. (34) are close to the fitted result listed in Table II. Therefore, the peak of mass quadrupole momentum $I_{22}^{m}$, as an important characteristic of the ringdown phase, is still qualitatively consistent with the prediction of PN theory.

Although Eq. (33) can predict the major pattern of $I_{22}^{m}-\left(\theta_{\text {init }}, \phi_{\text {init }}\right)$ relation, a correction term

$$
\sim \chi_{\text {init }}^{4} \sin ^{4} \theta_{\text {init }} f\left(\sin \phi_{\text {init }}, \cos \phi_{\text {init }}\right),
$$

is still needed if one wants to further recover subleading features. Here $f\left(\sin \phi_{\text {init }}, \cos \phi_{\text {init }}\right)$ is a function of $\phi_{\text {init }}$, corresponding to higher PN correction.

\section{E. The peak of current quadrupole wave $S_{22}^{m}$}

We now turn our attention to $S_{22}^{m}$. In Fig. 13, we use NRSur7dq4 and plot $S_{22}^{m}-\left(\theta_{\text {init }}, \phi_{\text {init }}\right)$ with $\chi_{\text {init }}=0.8$. The pattern is still symmetric about $\theta_{\text {init }}=\pi / 2$ and has a period $\pi$ in the $\phi_{\text {init }}$-direction, consistent with Eq. (27). We repeat our previous process and use PN predictions to understand the pattern. With PN theory, we have [118]

$$
\mathcal{S}_{22}=\mathcal{S}_{22}^{(1)}+\mathcal{S}_{22}^{(2)},
$$

where

$$
\begin{gathered}
\mathcal{S}_{22}^{(1)} \sim \chi_{\text {init }} \sin \theta_{\text {init }} r e^{-i \phi_{\text {init }}}, \\
\mathcal{S}_{22}^{(2)} \sim-\chi_{\text {init }} r v^{2} \sin \theta_{\text {init }} \cos \phi_{\text {init }} .
\end{gathered}
$$

Equations (37) lead to a fitting formula

$S_{22}^{m 2}=\chi_{\text {init }}^{2} \sin ^{2} \theta_{\text {init }}\left[Q^{(1)}+Q^{(2)} \sin 2\left(\phi_{\text {init }}+\phi_{S}\right)\right]$, 


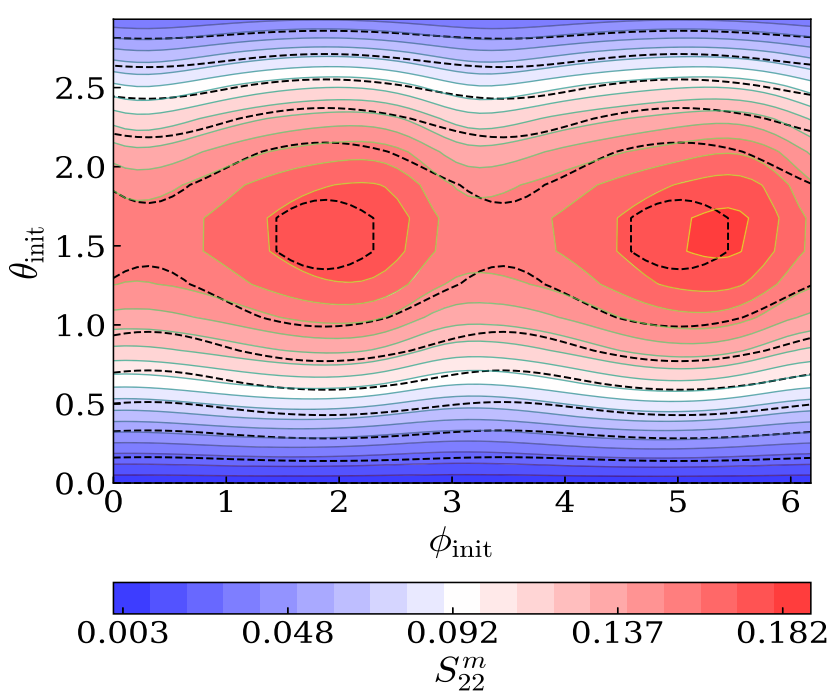

FIG. 13. The peak value of current quadrupole wave $S_{22}^{m}$ as a function of $\left(\theta_{\text {init }}, \phi_{\text {init }}\right)$, with $\chi_{\text {init }}=0.8$ (SKd configuration). The data are from NRSur7dq4, while dashed lines are the prediction of the PN-inspired counterpart in Eq. (38).

where $Q^{(1)}$ and $Q^{(1)}$ correspond to $\mathcal{S}_{22}^{(1)}$ and $\mathcal{S}_{22}^{(2)}$, respectively. The fitted value of $Q^{(2)}$ and $Q^{(1)}$ are $9.43 \times 10^{-3}$ and $4.28 \times$ $10^{-2}$. The ratio, $Q^{(2)} / Q^{(1)} \sim 0.22$, is close to $v^{2}$ at the light ring (0.33), which is again consistent with the PN prediction $Q^{(2)} / Q^{(1)} \sim v^{2}$. Therefore, the peak of current quadrupole wave $S_{22}^{m}$ also inherits information from the PN regime.

\section{F. The phase difference $\Delta \Phi_{\text {IS }}$}

We finally study the phase difference between the mass and current quadrupole waves $\Delta \Phi_{\mathrm{IS}}$, which is the key factor that determines the final kick velocity. Figure 14 is $\Delta \Phi_{\text {IS }}$ of the SKd4-"03" system (Table I). During the inspiral stage, $\Delta \Phi_{\text {IS }}$ accumulates monotonically over time. It then gradually settles down to a constant after the merger. In fact, one can use PN theory to understand the evolution of $\Delta \Phi_{\mathrm{IS}}$. Before the merger, we have [cf. Eqs. (18)]

$$
\Delta \Phi_{\mathrm{IS}}=\phi_{\mathrm{pre}}-\phi
$$

Here $\phi_{\text {pre }}$ is the precession phase of the spins, and is obtained by measuring the spins of each individual $\mathrm{BH} ; \phi$ is the orbital phase. In Fig. 14, we compare Eq. (39) to the NR result. Two results agree pretty well until $t \sim-50 M$. Near the merger, $\phi_{\text {pre }}$ is thought to be locked to $\phi$ [121], in order for the accumulation of $\Delta \Phi_{\text {IS }}$ to be halted. An alternative way to think of this is based on the QNM decomposition. For the ringdown portion of $I_{22}$ and $S_{22}$, they must both be decomposed into $(2,2)$ QNMs. After higher overtones decay away $(t>20 M)$, we are left with the fundamental mode [see Eq. (5) for more details]

$$
I_{22} \sim \mathcal{A}_{0}^{(I)} e^{i \varphi_{0}^{(I)}} e^{-i \omega_{220} t}, \quad S_{22} \sim \mathcal{A}_{0}^{(S)} e^{i \varphi_{0}^{(S)}} e^{-i \omega_{220} t},
$$

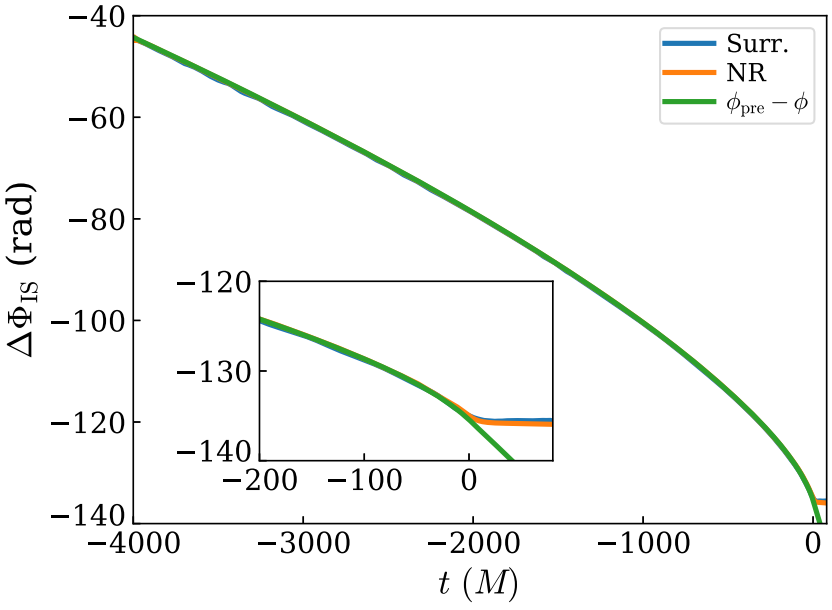

FIG. 14. The time evolution of $\Delta \Phi_{\text {IS }}$ for the SKd4-“03" system (orange curve). It is compared to NRSur7dq4 (blue curve) with the same initial condition. As expected, their results are close. Within the inspiral regime, PN theory predicts $\Delta \Phi_{\mathrm{IS}}=\phi_{\text {pre }}-\phi$, which is shown as the green curve.

which leads to $\Delta \Phi_{\mathrm{IS}}=\varphi_{0}^{(I)}-\varphi_{0}^{(S)}$, i.e., a constant. The fact that both $I_{22}$ and $S_{22}$ have the same QNM frequency is a consequence of the isospectrality feature of black holes.

Then we study how $\Delta \Phi_{\text {IS }}$ depends on the progenitor's parameters. We first choose eight NR runs in Table I, whose $\phi_{\text {init }}$ are different. As shown in Fig. $15, \Delta \Phi_{\text {IS }}$ with different

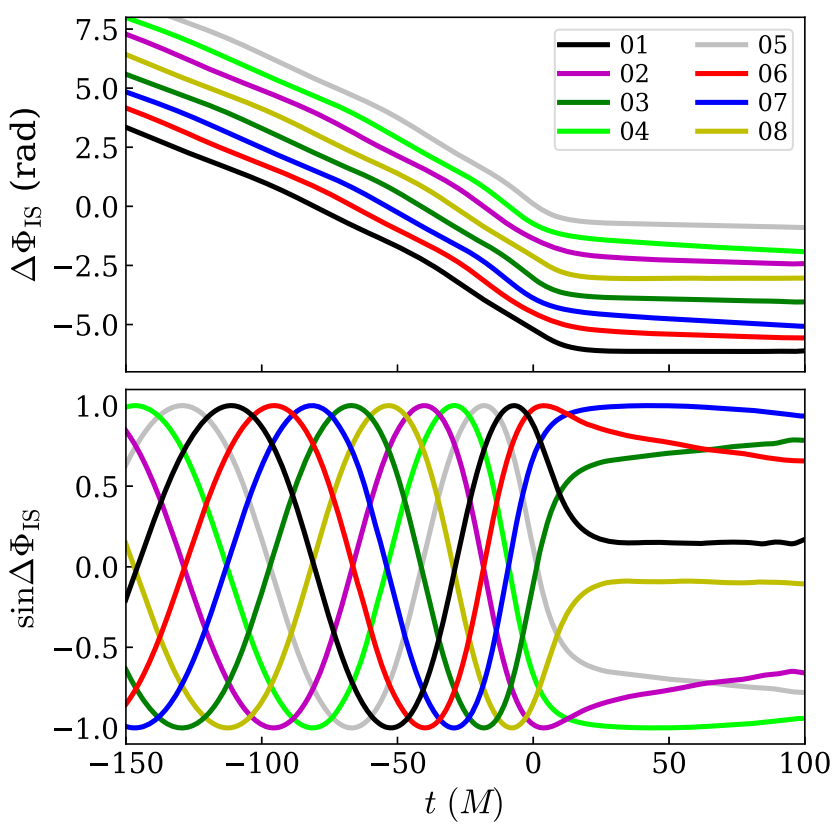

FIG. 15. The evolution of $\Delta \Phi_{\text {IS }}$ for SKd4 systems (Table I). Eight runs start with different $\phi_{\text {init }}$, and their $\Delta \Phi_{\text {IS }}$ are finally locked to different values. The bottom panel is $\sin \Delta \Phi_{\text {IS }}$. One can directly estimate the kick velocity from the final value of $\sin \Delta \Phi_{\mathrm{IS}}$, since the kick is roughly proportional to the integration of $\sin \Delta \Phi_{\text {IS }}$ [Eq. (17)]. As for high-kick cases, their $\Delta \Phi_{\text {IS }}$ change slowly during the late postmerger stage. This is due to the Doppler shift. 
TABLE III. The mass of remnant BHs inferred from $h_{22}$ and $h_{2,-2}$, by fitting with QNMs (7 overtones, see Sec. II for more details). Four runs below are in the SKu configuration (Table V) with $\chi_{\text {init }}=0.8$ and different $\phi_{\text {init }}$. Among them, SKu8-“02" and SKu8-“04" are high-kick cases. The masses inferred from $h_{22}$ and $h_{2,-2}$ are quite different, and the relative difference are close to the final kick velocity.

\begin{tabular}{|c|c|c|c|c|}
\hline Runs (SKu8) & “01” & “02” & “03” & “04” \\
\hline Mass from NR & 0.941 & 0.939 & 0.941 & 0.939 \\
\hline Mass from $h_{2,2}$ & 0.940 & 0.945 & 0.941 & 0.931 \\
\hline Mass from $h_{2,-2}$ & 0.940 & 0.931 & 0.940 & 0.945 \\
\hline Relative mass difference between $h_{2, \pm 2}$ & $-7.8 \times 10^{-5}$ & -0.015 & $3.3 \times 10^{-4}$ & 0.015 \\
\hline Final kick & $-1.6 \times 10^{-3}$ & -0.011 & $1.3 \times 10^{-3}$ & 0.011 \\
\hline
\end{tabular}

$\phi_{\text {init }}$ are finally locked to different values. The bottom panel is $\sin \Delta \Phi_{\text {IS }}$. Recalling that the kick velocity can be roughly estimated by integrating $\sin \Delta \Phi_{\text {IS }}$ [Eq. (17)], the final value of $\sin \Delta \Phi_{\mathrm{IS}}$ is a strong signature for the final kick velocity. For instance, one can directly read that SKd4-"07" leads to a positive largest kick, consistent with NR results (Table I). Interestingly, $\Delta \Phi_{\text {IS }}$ of several runs (e.g., "03") do not settle into a constant. Instead, there are slow changes over time. This is because the final BHs are boosted with relatively large kick velocities. As a result, there is a Doppler shift between the mode frequency of $h_{22}$ and of $h_{2,-2}$, recalling that $h_{22}$ is dominantly emitted upward, while $h_{2,-2}$ downward [96]. To test our statement, we pick four of SKu systems that are listed in Table V. Here we choose SKu systems since they lead to larger kicks, thus the comparison is less impacted by numerical noises. The results are summarized in Table III. We can see relative mass differences are close to the kick of final BHs. A slight difference in mass leads to a deviation between the mode frequency of $h_{22}$ and $h_{2,-2}$, i.e., [see Eq. (4)]

$$
\begin{gathered}
h_{22} \sim \mathcal{A}_{220} e^{i \psi_{220}} e^{-i \omega_{220}(1+\delta) t}, \\
h_{2,-2}^{*} \sim \mathcal{A}_{2,-20} e^{-i \psi_{2,-20}} e^{-i \omega_{220}(1-\delta) t},
\end{gathered}
$$

where $\delta$ is a small parameter, and is proportional to the kick velocity. In the late time regime, Eq. (41) implies

$$
\begin{aligned}
\sin \Delta \Phi_{\mathrm{IS}}= & \sin \left[\varphi_{0}^{(I)}-\varphi_{0}^{(S)}\right] \\
& +\frac{4\left(\mathcal{A}_{220}^{2}-\mathcal{A}_{2,-20}^{2}\right) \mathcal{A}_{220} \mathcal{A}_{2,-20}}{\left|\mathcal{A}_{220}^{2} e^{-i\left(\psi_{220}+\psi_{2,-20}\right)}-\mathcal{A}_{2,-20}^{2} e^{i\left(\psi_{220}+\psi_{2,-20}\right)}\right|^{2}} \\
& \times \cos \left(\psi_{220}+\psi_{2,-20}\right) \omega_{220} t \delta+\mathcal{O}\left(\delta^{2}\right) .
\end{aligned}
$$

The new term above gives rise to a linear change in time, and it is consistent with the feature which we observe in Fig. 15.

We then use NRSur7dq4 to explore more parameter space of SKd systems, by varying three free parameters $\chi_{\text {init }}, \theta_{\text {init }}$ and $\phi_{\text {init }}$, respectively. Results are shown in the bottom row of Fig. 10 . We can see that $\chi_{\text {init }}$ and $\theta_{\text {init }}$ do not affect $\Delta \Phi_{\text {IS }}$, even near and after the merger. Meanwhile, $\phi_{\text {init }}$ gives rise to only a constant phase shift for $\Delta \Phi_{\text {IS }}$, consistent with what we obtained in Sec. III C [Eq. (30)]. In fact, if we subtract $\phi_{\text {init }}$ from $\Delta \Phi_{\text {IS }}$, the rest of time dependence is still insensitive to $\phi_{\text {init }}$, although not as good as the cases of $\chi_{\text {init }}$ and $\theta_{\text {init }}$.

Recalling that the final kick velocity is given by [Eqs. (17) and (28)]

$$
\begin{aligned}
v_{f} & \sim \operatorname{Im} \int \dot{I}_{22} \dot{S}_{22}^{*} d t \sim I_{22}^{m} S_{22}^{m} \operatorname{Im} e^{i \phi_{\text {init }}} \int \dot{T}_{I}(t) \dot{T}_{S}^{*}(t) d t \\
& \sim \chi_{\text {init }} \sin \theta_{\text {init }} \sin \left(\phi_{\text {init }}-\phi_{\text {init }}^{(0)}\right),
\end{aligned}
$$

where we have used the leading terms in Eq. (33) and (38). This result is the same as Eq. (14), as discussed in [78-80]. To offer an illustration, we use NRSur7dq4Remnant to plot $v_{f}$ as a function of $\left(\theta_{\text {init }}, \phi_{\text {init }}\right)$ in Fig. 16 , with $\chi_{\text {init }}=0.76$. Meanwhile, we use Eq. (43) to fit the $v_{f}-\left(\chi_{\text {init }}, \theta_{\text {init }}, \phi_{\text {init }}\right)$ dependence, and the result is shown as dashed lines in Fig. 16. We can see Eq. (43) works properly.

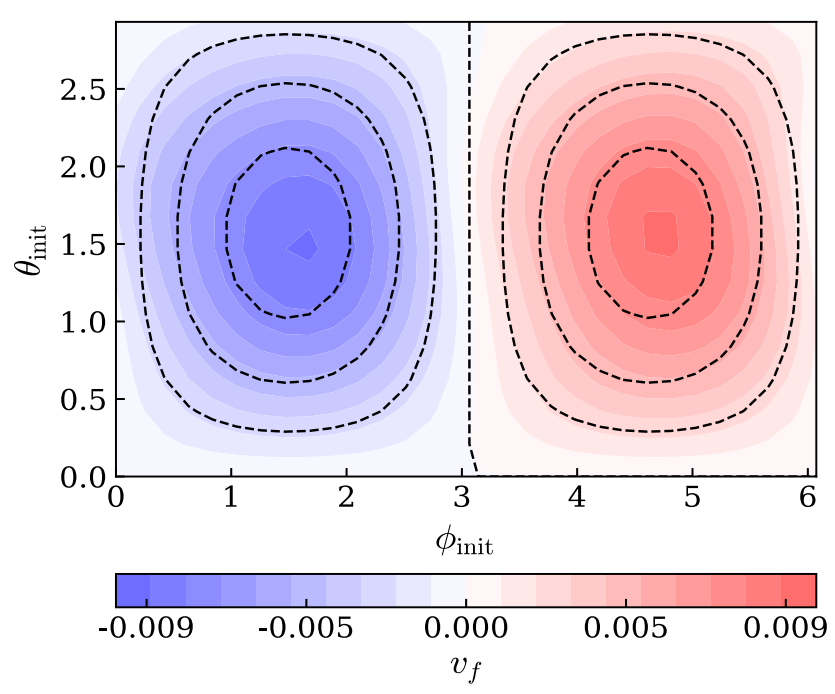

FIG. 16. The final kick velocity as a function of $\left(\theta_{\text {init }}, \phi_{\text {init }}\right)$, predicted by NRSur7dq4Remnant. The component spin $\chi_{\text {init }}$ is chosen to be 0.76 . The contours with dashed lines are from Eq. (43). 


\section{BACKWARDS ONE-BODY MODEL}

In this section, we shall focus on the time evolution of the mass and current quadrupole waves, $T_{I}(t)$ and $T_{S}(t)$, as defined in Eq. (28). In particular, we use an analytic phenomenological model BOB, conceived by McWilliams [111], to model the ringdown evolution. We first give a brief introduction to BOB in Sec. IV A, and then compare it to NR results in Sec. IV B.

\section{A. A brief review of $\mathrm{BOB}$}

The BOB model is an accurate, fully analytical GW waveform model for the late inspiral, merger and ringdown of $\mathrm{BBH}$ [111], which is able to match the waveform 20M before the peak of strain. This feature enables people to avoid the extrapolation of inspiral models beyond their domain of validity. Here we restrict our attention to the ringdown portion.

As discussed in Refs. [111,122], the amplitude of the News $\left|\dot{h}_{\ell m}\right|$ is related to its frequency $\Omega_{\ell m}$ by

$$
\left|\dot{h}_{\ell m}\right|^{2} \propto \frac{d}{d t} \Omega_{\ell m}^{2}
$$

where the coefficient remains (approximately) constant throughout the merger and ringdown phase. It was found ${ }^{5}$ that either $\left|h_{2, \pm 2}\right|,\left|\dot{h}_{2, \pm 2}\right|$, or $\left|\ddot{h}_{2, \pm 2}\right|$ can be modeled by

$$
X \operatorname{sech}\left[\gamma\left(t-t_{p}\right)\right]
$$

with two free variables $X$ and $t_{p}$, where $\gamma=-\operatorname{Im} \omega_{220}$ is the decay rate of the fundamental mode, determined by the final mass $m_{f}$ and spin $\chi_{f}$. Applying Eq. (45) to $\left|\ddot{h}_{2, \pm 2}\right|$, $\left|\dot{h}_{2, \pm 2}\right|$, and $\left|h_{2, \pm 2}\right|$ leads to three classes of BOB. Below we shall discuss the model for $\dot{h}_{2, \pm 2}$, and refer the interested reader to Appendix B for $\ddot{h}_{2, \pm 2}$ and $h_{2, \pm 2}$.

We first write the News $h_{22}$ as

$$
\dot{h}_{22}=X \operatorname{sech}\left[\gamma\left(t-t_{p}\right)\right] e^{-i \phi_{22}(t)},
$$

where $X$ is essentially the peak value of $\left|\dot{h}_{22}\right|$, and $t_{p}(>0 M)$ is its peak time. Using the relation in Eq. (44), we obtain

$\dot{\phi}_{22}(t)=\Omega_{22}(t)=\left\{\Omega_{0}^{2}+\frac{\omega_{0}^{2}-\Omega_{0}^{2}}{2}\left[\tanh \gamma\left(t-t_{p}\right)+1\right]\right\}^{1 / 2}$,

where $\Omega_{0}$ is an integration constant and $\omega_{0}=\operatorname{Re} \omega_{220}$. Equation (47) indicates that

\footnotetext{
${ }^{5}$ In Ref. [111], the author pointed out that this phenomenological formula works best for $\left|\ddot{h}_{\ell m}\right|$. For now, we try to make our statement general, and make comparisons later.
}

$$
\lim _{t \rightarrow \infty} \dot{\phi}_{22}(t)=\omega_{0}
$$

i.e., $\dot{h}_{22}$ oscillates at the fundamental QNM frequency during the late time of postmerger portion. Integrating Eq. (47) again gives

$$
\phi_{22}=\frac{1}{\gamma}\left(\omega_{0} \operatorname{arctanh} \frac{\dot{\phi}_{22}}{\omega_{0}}-\Omega_{0} \operatorname{arctanh} \frac{\Omega_{0}}{\dot{\phi}_{22}}\right)-\phi_{0},
$$

where $\phi_{0}$ is another integration constant. We can see that $\dot{h}_{22}$ depends on 6 parameters

$$
X, m_{f}, \chi_{f}, t_{p}, \Omega_{0}, \phi_{0} .
$$

Similarly, Eqs. (46)-(49) can also be applied to $\dot{h}_{2,-2}^{*}, \dot{I}_{22}$, and $\dot{S}_{22}$.

As $t \gg t_{p}$, we obtain an asymptotic expansion for $\dot{h}_{22}$

$$
\dot{h}_{22}=\left(2 X e^{\gamma t_{p}}\right) e^{i \psi_{0}} e^{-i \omega_{220} t},
$$

where

$\psi_{0}=\omega_{0} t_{p}+\phi_{0}+\frac{\Omega_{0}}{\gamma} \operatorname{arctanh} \frac{\Omega_{0}}{\omega_{0}}-\frac{\omega_{0}}{\gamma} \frac{1}{2} \log \frac{4 \omega_{0}^{2}}{\omega_{0}^{2}-\Omega_{0}^{2}}$.

By comparing Eq. (51) with the overtone decomposition [e.g., Eq. (4)], $\left(2 X e^{\gamma t_{p}}\right)$ is supposed to be equal to $\left|\omega_{220} \mathcal{A}_{220}\right|$.

\section{B. Numerical comparisons}

In this subsection, we use our NR simulations (Tables I and V) to study the accuracy of BOB. To begin with, we take the ringdown portion of SKd4-"03" (Table I), and fit $\dot{h}_{2, \pm 2}, \dot{I}_{22}, \dot{S}_{22}$ to Eq. (46), respectively. Similar to the previous QNM fitting algorithm (Sec. II), we fit $X$ and $\phi_{0}$ with unweighted linear least squares, and fit $m_{f}, \chi_{f}, t_{p}, \Omega_{0}$ with nonlinear least squares. To give a comparison, we also fit the ringdown sector with QNMs. As shown in Fig. 17, the BOB can capture the major feature of $\dot{h}_{2, \pm 2}, \dot{I}_{22}, \dot{S}_{22}$. Their residuals are all on the order of $\sim 10^{-3}$, an order of magnitude worse than the fitting of QNMs. Note that there are fewer free parameters for the fitting of $\mathrm{BOB}$ than the QNM decomposition, a fairer comparison would be restricting to only 2 QNMs (so that there are 6 free parameters for both models) and studying the late ringdown portion. ${ }^{6}$ This is beyond the scope of this paper, and we leave the relevant discussions for future study. Table IV is a summary for the fitting results, where the last row is the mismatch between $\mathrm{BOB}$ and NR, defined by

\footnotetext{
${ }^{6}$ Based on Table I of Ref. [30], it corresponds to $\sim 20 M$ after the peak of strain.
} 

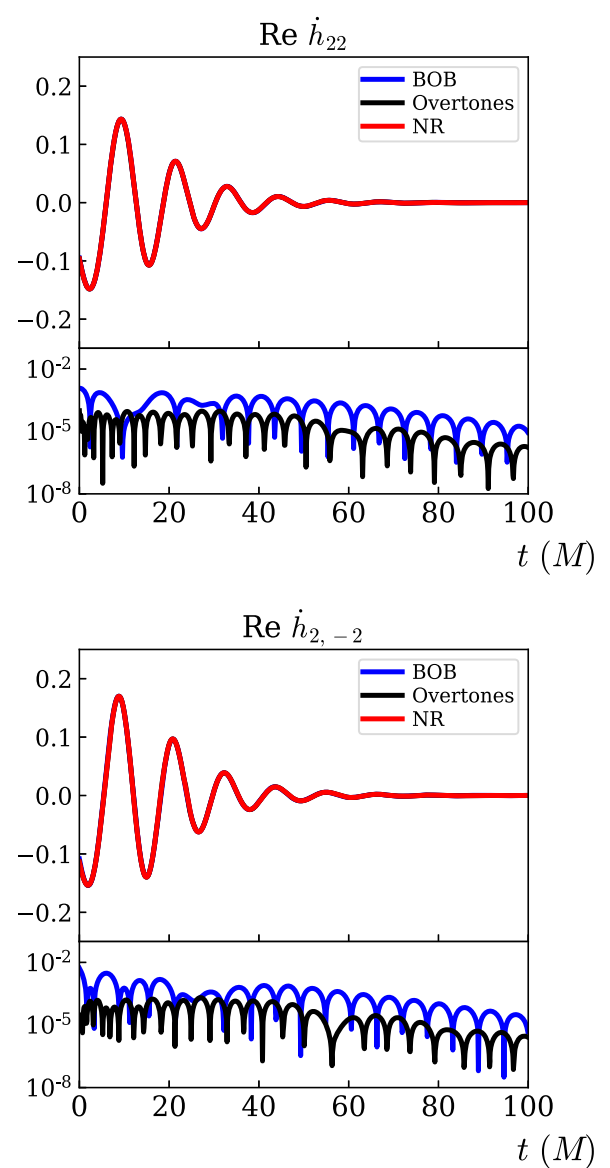
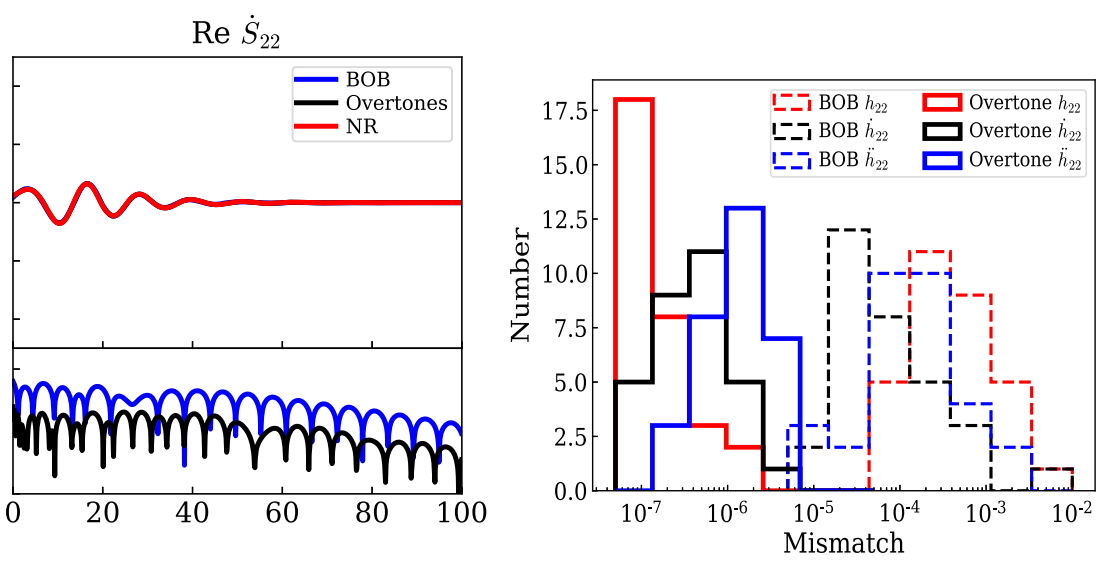

$\operatorname{Re} \dot{I}_{22}$
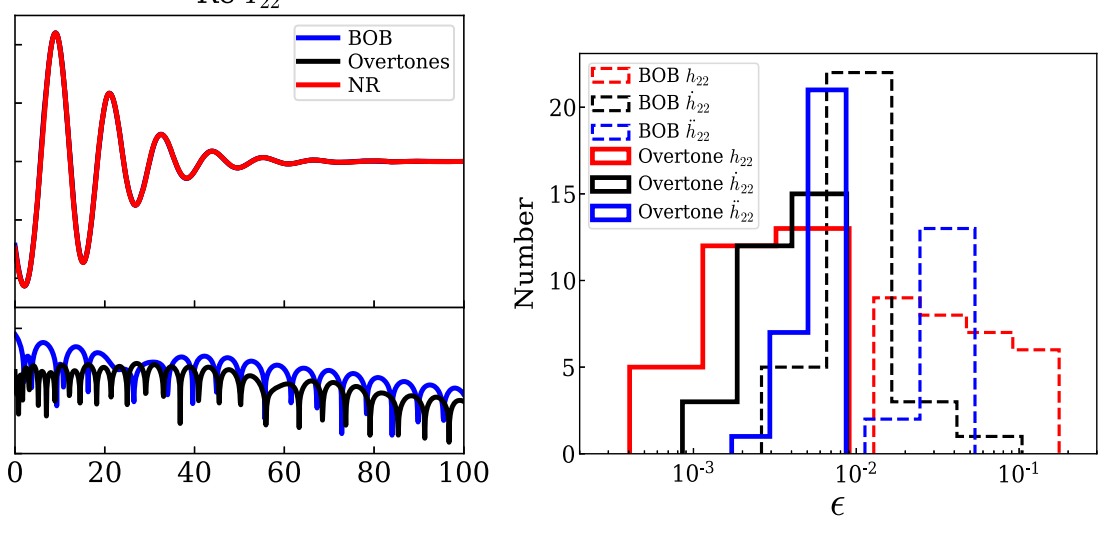

FIG. 17. The BOB model for $\dot{h}_{22}, \dot{h}_{2,-2}, \dot{I}_{22}$, and $\dot{S}_{22}$ (the left and middle columns). They are compared to the ringdown portion of SKd4-“03". We also fit data with QNMs. The residuals of BOB for four variables are all on order of $\sim 10^{-3}$, an order of magnitude worse than the fitting of QNMs. The right column corresponds to the distribution of mismatch [top panel, see Eq. (53)], and parameter deviation [bottom panel, see Eq. (55)] for QNM decomposition and BOB, using our NR simulations listed in Tables I and V. BOB is always worse than QNM fitting.

$$
\text { Mismatch }=1-\frac{\left(h_{\mathrm{B}}, h_{\mathrm{NR}}\right)}{\sqrt{\left(h_{\mathrm{NR}}, h_{\mathrm{NR}}\right)\left(h_{\mathrm{B}}, h_{\mathrm{B}}\right)}},
$$

with

$$
\left(h_{\mathrm{B}}, h_{\mathrm{NR}}\right)=\operatorname{Re} \int_{0 M}^{100 M} h_{\mathrm{B}} h_{\mathrm{NR}}^{*} d t,
$$

where $h_{\mathrm{B}}$ and $h_{\mathrm{NR}}$ are the complex strains of BOB and NR in the time domain, respectively. The integration limit is taken to be the ringdown sector. We can see that the BOB for $\dot{I}_{22}$ and $\dot{h}_{22}$ lead to smaller mismatches than the other two. Meanwhile, the BOB behaves worst for $\dot{S}_{22}$ : even though the mismatches can reach $2 \times 10^{-3}$, this is much higher than those achievable by $\dot{I}_{22}$ and $\dot{h}_{22}$; furthermore, the recovered estimations for spin and mass of the final black hole are substantially biased.

We then use Eq. (51) to make a connection between BOB and QNM decomposition, i.e., expanding BOB for
TABLE IV. Fitting $\dot{h}_{22}, \dot{h}_{2,-2}, \dot{I}_{22}, \dot{S}_{22}$ to the BOB model, respectively. The original data is the ringdown portion of SKd4"03". The first four rows are the free parameters of BOB: peak magnitude $X$, peak time $t_{p}$, final spin $\chi_{f}$, and final mass $m_{f}$. Comparing with the NR prediction of final spin (0.685) and final mass (0.951), the BOB for $\dot{I}_{22}$ and $\dot{h}_{22}$ are more accurate to recover the final properties than the other two. The model for $\dot{S}_{22}$ is the worst. Using the BOB's asymptotic expansion in the late time limit [Eq. (51)], $2 X e^{\gamma t_{p}}$ (the sixth row) is expected to be equal to $\left|\omega_{220} \mathcal{A}_{220}\right|$ (the seventh row). The agreement for $\dot{S}_{22}$ is the worst. The last row is the mismatch between BOB and the original NR data.

\begin{tabular}{llllc}
\hline \hline & $\dot{h}_{22}$ & $\dot{h}_{2,-2}$ & $\dot{I}_{22}$ & $\dot{S}_{22}$ \\
\hline$X$ & 0.153 & 0.171 & 0.227 & 0.035 \\
$t_{p} / M$ & 5.13 & 7.31 & 6.21 & 12.53 \\
$\chi_{f}$ & 0.684 & 0.681 & 0.686 & 0.559 \\
$m_{f} / M$ & 0.954 & 0.944 & 0.951 & 0.857 \\
$2 X e^{\gamma t_{p}}$ & 0.474 & 0.644 & 0.771 & 0.247 \\
$\left|\omega_{220} \mathcal{A}_{220}\right|$ & 0.470 & 0.622 & 0.759 & 0.175 \\
Mismatch $\left(\times 10^{-5}\right)$ & 2.6 & 9.9 & 3.1 & 204.0 \\
\hline \hline
\end{tabular}


the late-time ringdown $\left(t \gg t_{p}\right)$. The value of $\left(2 X e^{\gamma t_{p}}\right)$ is expected to be close to $\left|\omega_{220} \mathcal{A}_{220}\right|$, so we make such a comparison in the sixth and seventh rows of Table IV. We can see that $\dot{h}_{22}$ leads to the best agreement, while $\dot{S}_{22}$ the worst.

As we mentioned earlier, $X \operatorname{sech}\left[\gamma\left(t-t_{p}\right)\right]$ can also be used to describe the magnitude of $\ddot{h}_{2, \pm 2}$ or $h_{22}$. Each of them leads to a class of BOB model (see Appendix B for more details). We study their accuracy by fitting our simulations (cf. Table I and V) to those three classes of $\mathrm{BOB}$, and showing the distribution of mismatches (with NR waveforms) in the third column of Fig. 17. Generally speaking, the mismatches of $\mathrm{BOB}$ are $10^{-5}-10^{-2}$, which are worse than those of QNM decomposition. Among the three classes, $\ddot{h}_{22}$ gives the smallest mismatch, while $h_{22}$ the largest.

Another way to quantify the accuracy of $\mathrm{BOB}$ is to compare the inferred spin and mass (from the fitting) to NR predictions. Similar to Ref. [30], we define a parameter deviation

$$
\epsilon=\sqrt{\left(\delta M_{f} / M\right)^{2}+\left(\delta \chi_{f}\right)^{2}}
$$

and plot its distribution in Fig. 17. We can see $\epsilon$ for BOB is also worse than the QNM decomposition. In addition, the distribution of $\epsilon$ show that the BOB works worst for $h_{22}$, and best for $\dot{h}_{22}$.

\section{PARAMETER-ESTIMATION CONTRIBUTIONS FROM INSPIRAL AND RINGDOWN STAGES}

In this section, we demonstrate, with a few example sources, the impact of the ringdown portion of the waveforms - as well as the correlation between the ringdown and the inspiral phases-to parameter estimation errors. To do this, we will apply the Fisher-matrix formalism to the NRSur7dq4 surrogate waveforms (for BBHs with $1<q<4$ and individual dimensionless spin $\chi<0.8)[54,55]$,

In Sec. VA, we will give a brief review for the Fishermatrix formalism. In Sec. V B, we discuss nonprecessing $\mathrm{BBH}$ systems with varying total mass, illustrating how information contribution from the ringdown stage gains more importance for more massive systems. Finally, in Sec. V C, we study parameter estimation errors of precessing systems, illustrating how estimations of individual spin components will benefit from information from the ringdown stage.

\section{A. The Fisher-matrix formalism and waveform models}

For a gravitational waveform $h\left(\theta^{j}\right)$ that depends on a list of parameters $\theta^{j}$, the Fisher matrix is given by

$$
\Gamma_{i j}=\left(\frac{\partial h}{\partial \theta^{i}} \mid \frac{\partial h}{\partial \theta^{j}}\right) .
$$

Here the inner product between two waveforms $(h \mid g)$ is defined as

$$
(h \mid g)=4 \operatorname{Re} \int \frac{\tilde{h}^{*}(f) \tilde{g}(f)}{S_{n}(f)} d f,
$$

with the superscript $*$ standing for complex conjugation, and $S_{n}(f)$ the spectral density of the noise when detecting $h$. In terms of this inner product, the signal-to-noise ratio (SNR) of a signal $h$ is given by $\sqrt{(h \mid h)}$.

The covariance matrix for the estimated values of $\theta^{j}$, in presence of noise, is given by the inverse of the Fisher matrix,

$$
\operatorname{Var}\left(\theta^{i}, \theta^{j}\right)=\left(\Gamma^{-1}\right)_{i j}
$$

From this, we obtain the individual estimation error for $\theta^{j}$,

$$
\Delta \theta^{i}=\sqrt{\left(\Gamma^{-1}\right)_{i i}}
$$

and the correlation coefficient between $\theta^{i}$ and $\theta^{j}$,

$$
\operatorname{Corr}\left(\theta^{i}, \theta^{j}\right)=\frac{\left(\Gamma^{-1}\right)_{i j}}{\sqrt{\left(\Gamma^{-1}\right)_{i i}\left(\Gamma^{-1}\right)_{j j}}} .
$$

Waveforms described by the NRSur7dq4 surrogate model are parametrized by 13 parameters:

$$
\chi_{1 z}, \chi_{1 p}, \phi_{1}, \chi_{2 z}, \chi_{2 p}, \phi_{2}, M, q, l, \beta, t_{c}, \phi_{c}, D .
$$

Correspondingly, we have a 13-dimensional Fisher matrix. Here, the subscripts "1" and " 2 " stand for the two individual black holes in the binary system, $\chi_{z}$ is the spin component in the direction of orbital angular momentum, $M$ is the total mass in the detector frame, $q>1$ is the mass ratio, $D$ is the luminosity distance between the source and the detector, and $l$ and $\beta$ describe the wave emission direction in the frame of the source. The spin component in the orbital plane is parametrized by the magnitude $\chi_{p}$ and the azimuthal angle $\phi$. Finally, $t_{c}$ and $\phi_{c}$ are the coalescence time and phase, respectively.

Throughout this paper, we adopt the Ansatz that the two gravitational-wave polarizations, $h_{+}$and $h_{\times}$, can be individually measured, both with the noise spectrum $S_{n}$. This simplification allows us not to explicitly include sky location and orientation of the source; it can be justified in the situation of a three-detector network that can provide good source localization. In this way, results given in this section should be more optimistic than the actual situation.

\section{B. Inspiral versus ringdown: Nonprecessing binaries}

In this paper, we will focus mainly on the Cosmic Explorer (CE) [123], whose $S_{n}(f)$ is shown in Fig. 18. 


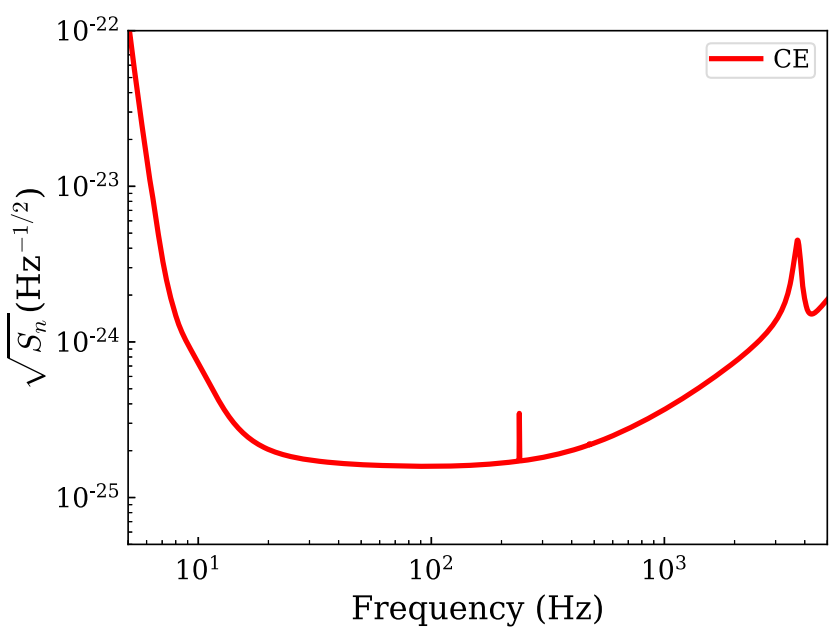

FIG. 18. The noise spectral density of Cosmic Explorer.

Using this sensitivity, in Fig. 19, we show the SNR of an optimally oriented $\mathrm{BBH}$ with varying total (detector-frame) mass $M$ and mass ratio $q$, assuming the system is at redshift $z=1\left(D_{L}=6.7 \mathrm{Gpc}\right)$. Note that the intrinsic total mass $M$. is given by $M /(1+z)$. The high SNR shown in this figure indicates that in the $3 \mathrm{G}$ era, we will most frequently be detecting binaries at cosmological distances of around $1 \lesssim z \lesssim 3$. Correspondingly, we will be observing these binaries with higher detector-frame masses, with factor 2 to 4 . In this way, events like GW150914 can be redshifted to around $\sim 130-260 M_{\odot}$, while heavy binaries like GW190521 can be shifted to 302-604 $M_{\odot}$. In the rest of this section, we shall study BBH systems with increasing

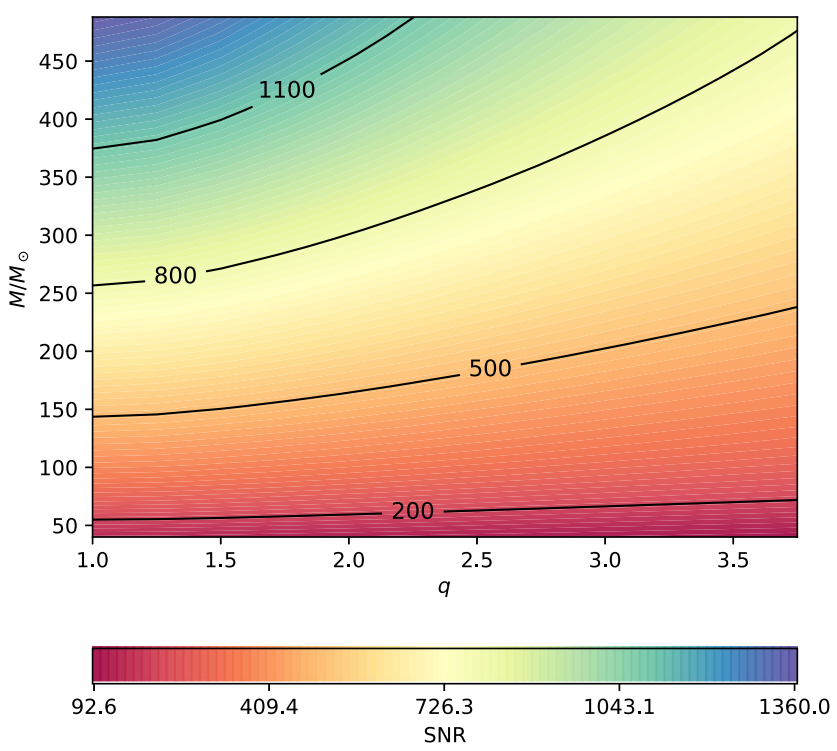

FIG. 19. The SNR of an optimally oriented GW event with varying total (detector-frame) mass $M$ and mass ratio $q$, assuming the system is at redshift $z=1(6.7 \mathrm{Gpc})$ and using $S_{n}(f)$ of the CE. total mass, in order to observe the increased importance of information contribution from the ringdown stage.

In order to study the ringdown and the inspiral portions individually, we separate two sectors (in the frequency domain) with the instantaneous $h_{22}$ frequency at $t=0 M$ (where $\sqrt{\sum_{l, m}\left|h_{l m}\right|^{2}}$ is maximum). For nonprecessing binaries, we will fix $q=2.3, \chi_{1 z}=0.1, \chi_{2 z}=0.6$, $\chi_{1 p}=\chi_{2 p}=0, \quad l=3 \pi / 10, \quad \beta=\pi / 2, \quad$ and consider $M / M_{\odot}=160,250,340$. For comparison purposes, we normalize all waveforms so that the SNR of the entire waveform is 200. We consider joint parameter estimations errors of $\left(\chi_{1 z}, \chi_{2 z}\right)$ and $(M, q)$, with results shown in Fig. 20 (blue for inspiral alone, black for ringdown alone, and red for combined). As a reference, we also list the SNR of each sector in the figure (cf. numbers in parentheses).

Regarding the overall size of the error ellipses, for the BBH system with total mass $M=160 M_{\odot}$, constraints from the ringdown sector are worse than those from the inspiral portion. As $M$ increases to $250 M_{\odot}$, constraints from the two sectors become comparable. For more
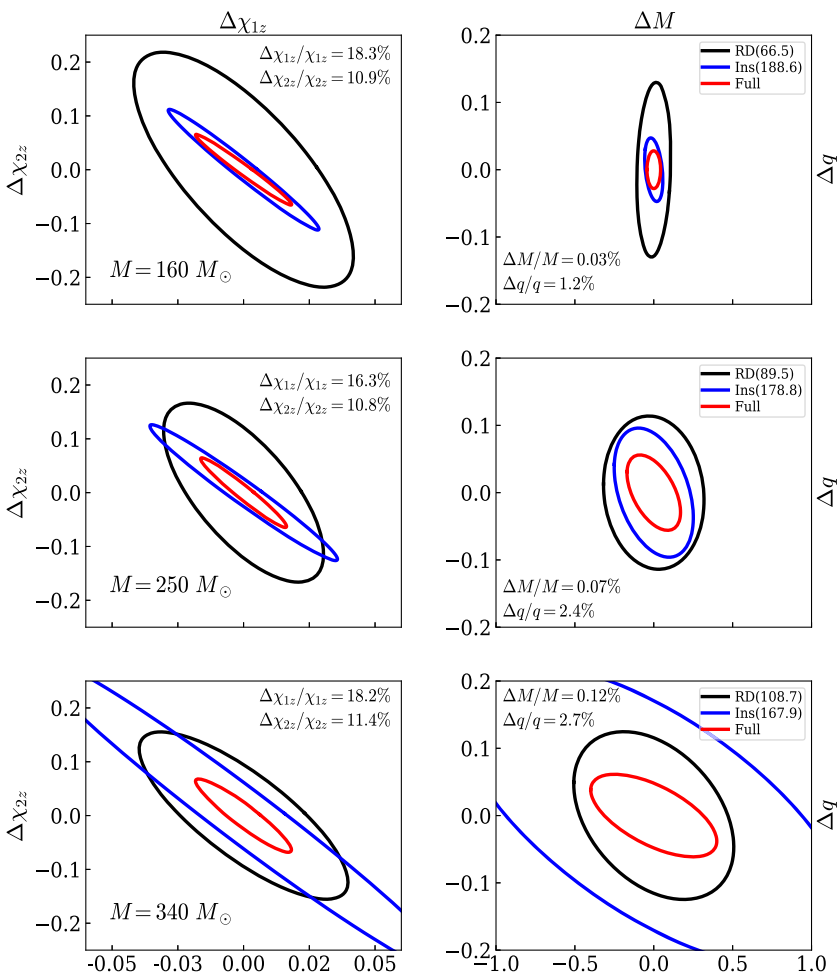

FIG. 20. The error ellipses of $\chi_{1 z}$ and $\chi_{2 z}$ (the left column), as well as $M$ and $q$ (the right column), using the data from NRSur7dq4. Two individual spins are both aligned with the orbital angular momentum, and other parameters are $q=2.3$, $\chi_{1 z}=0.1, \chi_{2 z}=0.6, \imath=3 \pi / 10, \beta=\pi / 2, \chi_{1 p}=\chi_{2 p}=0$. The total mass is chosen to be $160 M_{\odot}$ (the upper row), $250 M_{\odot}$ (the middle row) and $340 M_{\odot}$ (the bottom row). Three colors stand for the ringdown (black), inspiral (blue), and full sector (red), respectively. The numbers in parentheses are SNRs, where we normalize the total SNR of each event to 200 for comparison. 

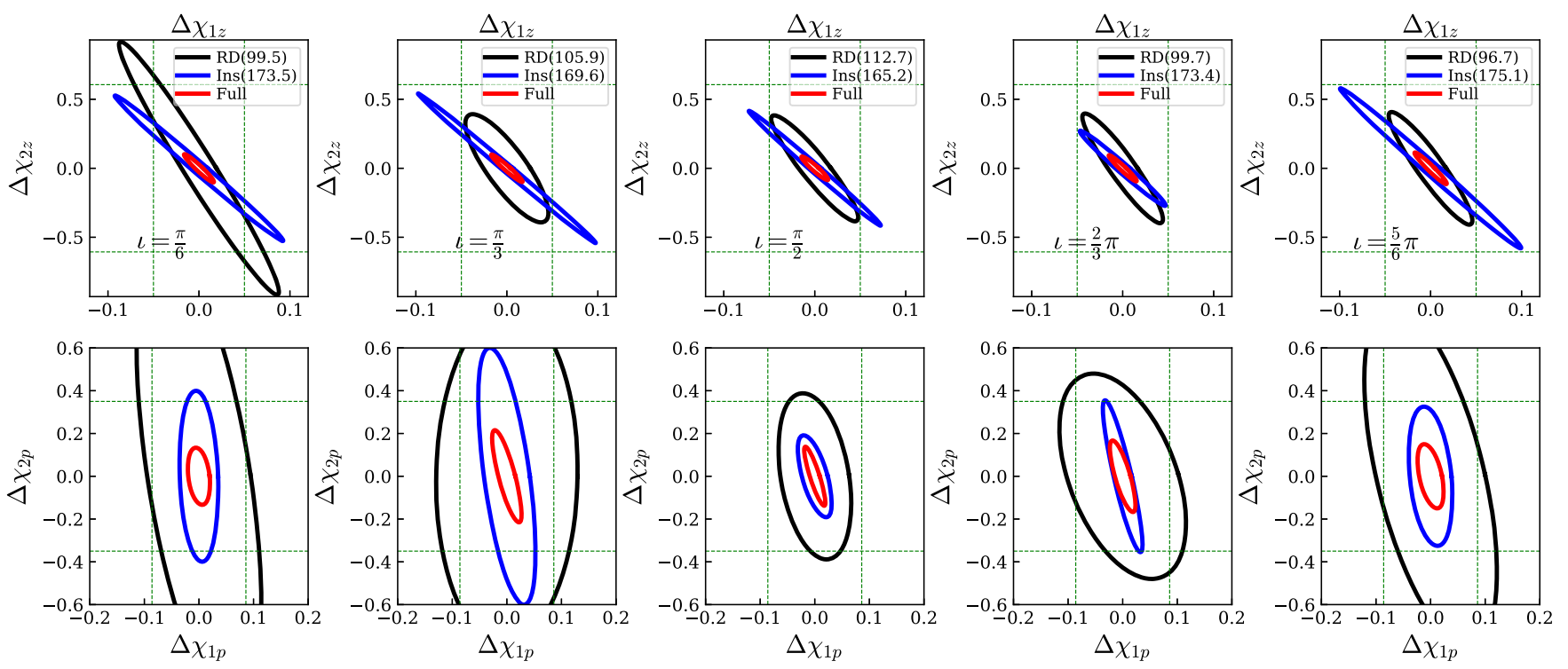

FIG. 21. Similar to Fig. 20, the error ellipses of $\chi_{1 z}$ and $\chi_{2 z}$ (the first row), and $\chi_{1 p}$ and $\chi_{2 p}$ (the second row), with different $l$ (each column). The green dashed lines stand for the original value of each parameter. Thus we have a meaningful measurement $(<100 \%)$ on a parameter if the error ellipse is within the dashed lines. The BBH systems have parameters $M=300 M_{\odot}, q=3.5, \chi_{1 z}=0.05$, $\chi_{1 p}=0.086, \chi_{2 z}=0.606, \chi_{2 p}=0.35, \phi_{1}=\pi / 13, \phi_{2}=43 \pi / 52, \beta=\pi / 2$. The error ellipses of ringdown and inspiral portions are not in the same direction, which implies different parameter correlations. After including the information of ringdown, the measurement accuracy of $\chi_{z}$ is improved by a factor of $\sim 4-5$, whereas $\chi_{p}$ is improved by a factor of $\sim 1.4$.

massive systems, the ringdown portion begins to dominate. It is remarkable that substantial parameter estimation can already be obtained from ringdown alone: this means not only the quasinormal mode frequency, but also the excitation amplitudes, are providing the information $[24,119,120,124,125]$. We also note that detector-frame mass of $250 M_{\odot}$ corresponds to intrinsic total mass of $\sim 125 M_{\odot}$ at $z=1$, which will not be a rare type of event in third-generation detectors.

For spin measurements, both ringdown and inspiral sectors lead to somewhat degenerate measurements of $\chi_{1 z}$ and $\chi_{2 z}$. In particular, the inspiral stage accurately measures the $\sim\left[q \chi_{1 z}+(1-q) \chi_{2 z}\right]$ direction (as can be argued from PN treatments [126]), while the ringdown has a less degenerate measurement, although with a most accurately measured combination similar to that from the inspiral. As for mass measurements, the ringdown and inspiral sectors lead to $M-q$ error ellipses with different directions, but no substantial degeneracy breaking.

\section{Precessing BBH systems}

We now turn our attention to precessing systems. We set the total mass of the systems to $300 M_{\odot}$, in order to make the contribution of ringdown sector comparable to the inspiral portion. Meanwhile, we choose $\chi_{1 z}=0.05, \chi_{2 z}=$ $0.606, \chi_{1 p}=0.086, \chi_{2 p}=0.35, \phi_{1}=\pi / 13, \phi_{2}=43 \pi / 52$. The observation is made at $\beta=\pi / 2$, with varying $l$.

We first study a BBH system with $q=3.5$. The results are shown in Fig. 21. We can see the relative size of ringdown and inspiral ellipses change with $t$. This is because the weights of different $\mathrm{GW}$ modes $h_{\ell m}$, i.e., ${ }_{-2} Y_{\ell m}(l, \beta)$, are functions of $l$. At different observational locations, the contributions from different GW modes $h_{\ell m}$ are different. Secondly, the $\chi_{1 z}-\chi_{2 z}$ error ellipse computed from the ringdown portion is tilted relative to that of inspiral sector, which implies that the parameter correlation of these two sectors are different. After combining the information of ringdown and inspiral, the measurement accuracy of $\chi_{z}$ is around 30\%, improved by a factor of $\sim 4-5$ compared with using the inspiral signal only. This result agrees qualitatively with the discussion in Ref. [14]. We note that SNR is 1.14 times greater after incorporating the ringdown signal. Hence most of the improvement is contributed from the correlation between the inspiral and ringdown, which leads to reduction of parameter degeneracy. On the other hand, the measurement accuracy of $\chi_{p}$ is only improved by a factor of $\sim 1.4$, not as good as the one of $\chi_{z}$. Nevertheless, the fractional error of $\chi_{p}$ is smaller than $100 \%$, hence we can still put meaningful constraints on $\chi_{p}$.

We want to remark that values mentioned above depend heavily on properties of the BBH system in question. For instance, for a low-mass-ratio BBH system with $q=1.2$, as shown in Fig. 22, the $\chi_{1 z}-\chi_{2 z}$ error ellipses computed from individual inspiral and ringdown sectors point along more similar directions. This leads to much less degeneracy breaking between $\chi_{1 z}$ and $\chi_{2 z}$ than in the $q=3.5$ case above, in particular making the measurement error $\Delta \chi_{1 z}$ 

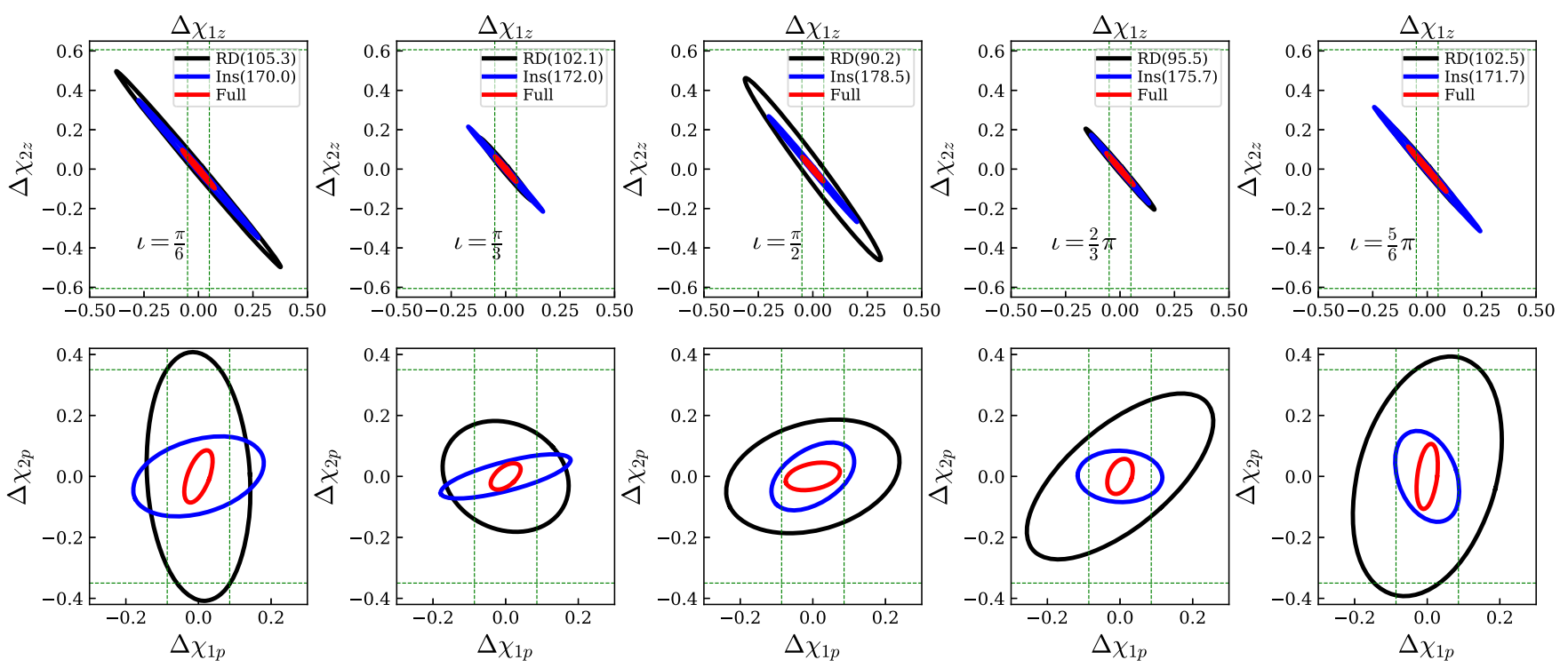

FIG. 22. Same as Fig. 21, except $q=1.2$.

greater than the value of $\chi_{1 z}$. Even so, incorporating ringdown, in addition to inspiral improves $\Delta \chi_{z} / \chi_{z}$ a factor of 2.8, substantially greater than the SNR improvement factor of around 1.16.

\section{CONCLUSIONS}

In this paper, we studied the gravitational waveforms of SKd systems, using both NR simulations (SpEC) and surrogate models (NRSur7dq4, NRSur7dq4Remnant). We first decomposed the ringdown portion of GW signal into QNMs, and explored how mode amplitudes of overtones depend on the progenitor's parameters (for $I_{22}, S_{22}$, as well as $h_{2 \pm 2}$ contents). We then studied the features of the mass and current quadrupole waves, focusing on their time evolutions and peak values. This leads to a qualitative understanding of kick velocity. Next, we fitted the evolution of $I_{22}(t)$ and $S_{22}(t)$ to the Backward-One-Body (BOB) model. Finally, we used Fisher information matrix to study the role of the ringdown state in parameter correlation. Here we summarize our main results:

(i) For SKd systems, the dependences of $I_{22}$ and $S_{22}$ on angular parameters $\left(\chi_{\text {init }}, \theta_{\text {init }}, \phi_{\text {init }}\right)$ can be separated from their temporal dependences [Eq. (28)].

(ii) (ii) Similar to the case of EMRI [105], the QNM amplitudes of SKd systems encode the information of progenitors' parameters. As an extension to Ref. [105], we included more overtones to the QNM decomposition. We found that the spectra peak at the fourth overtone, and that the dependence of mode magnitudes on $\phi_{\text {init }}$ is insensitive to the overtone index $n$ (up to a scaling factor). We found that the dependence of mode amplitudes on progenitor parameters is more easily understood when decomposed into mass and current quadrupole waves, instead of $(2,2)$ and $(2,-2)$ modes. (iii) Peak values of mass $\left(I_{22}^{m}\right)$ and current $\left(S_{22}^{m}\right)$ quadrupole waves encode the information of progenitors' spin. Enforced by the parity symmetry, the $\left(I_{22}^{m}, S_{22}^{m}\right)-$ $\left(\chi_{\text {init }}, \theta_{\text {init }}, \phi_{\text {init }}\right)$ pattern is symmetric about $\theta_{\text {init }}=$ $\pi / 2$ axis and has a period of $\pi$ in the direction of $\phi_{\text {init }}$-axis. Quantitatively speaking, the $\left(I_{22}^{m}, S_{22}^{m}\right)-$ $\left(\chi_{\text {init }}, \theta_{\text {init }}, \phi_{\text {init }}\right)$ dependence are consistent with the PN-inspired formulas.

(iv) The phase difference between mass and current quadrupole waves $\Delta \Phi_{\text {IS }}$ can lead to a qualitative understanding of kick velocity. Its time evolution can be anticipated from PN and black-hole perturbation theories: in the inspiral regime, $\Delta \Phi_{\text {IS }}$ is equal to the difference between the orbital and precession phases; near the merger, the spin precession rate is gradually locked to the orbital frequency-until well into the ringdown regime, when $\Delta \Phi_{\mathrm{IS}}$ should become constant since both $I_{22}$ and $S_{22}$ oscillate at the fundamental QNM frequency. However, we found that $\Delta \Phi_{\text {IS }}$ does not always settle down to a constant value during the postmerger stage, especially for high-kick cases. Instead, there is a slow change over time. This is due to the Doppler shift caused by the kick. The QNM frequency of $h_{22}$ (emitted upwards) is slightly different from the one of $h_{2,-2}$ (emitted downwards), which leads to a slow time evolution. In fact, the relative frequency difference is on the same order as the kick velocity.

(v) We verified that the BOB phenomenological model is accurate for the ringdown evolution of $\dot{h}_{2, \pm 2}$, $\ddot{h}_{2, \pm 2}$, and $I_{22}$, but much less so for $S_{22}$ and $h_{2, \pm 2}$. This calls for further, qualitative improvements of the current-quadrupole sector of the BOB model.

(vi) We found that in $3 \mathrm{G}$ detectors, the contribution of the ringdown part dominates over the inspiral 
part as the total detector-frame mass exceeds $\gtrsim 250-300 M_{\odot}$. We found that, as we combine both parts, the improvement in parameter estimation error is larger than the increase in SNR, indicating that the reduction of degeneracy due to the additional ringdown signal is the main reason for such improvement. As for $\chi_{z}$, in our examples, incorporating the information from ringdown signal can lead to $\sim 4-5$ times improvement on the measurement accuracy, while the accuracy for $\chi_{p}$ is improved by a factor of $\sim 1.4$.

Our results indicate that the ringdown sector of a $\mathrm{BBH}$ event encodes plenty of information about the progenitor. It also plays a complementary role to PN theory in the study of BBH evolution. In our study, we primarily focused on the SKd configuration. Future work could include more generic BBH systems and other GW modes, which can lead to more comprehensive understandings of the ringdown signals. Another possible avenue for future work is to increase the precision of NR surrogate models for the ringdown sector, since our work has revealed that the current NR surrogate models are not accurate enough for BH spectroscopy. A more accurate ringdown surrogate model will be beneficial for both data analysis and theoretical studies.

Meanwhile, as revealed in Fig. 5, as well as Eqs. (7) and (10), it might also be interesting for future work to investigate the features of mass and current quadrupole waves of EMRIs, which may turn out to be simpler than features found in Refs. [105-107]. Those further explorations could potentially provide us more physical understandings of EMRI ringdown spectra.

\section{ACKNOWLEDGMENTS}

We want to thank Serguei Ossokine, Alvin Chua, Gregorio Carullo, and Sean McWilliams for useful discussions. S. M. and Y. C. are supported by the Simons Foundation (Grant No. 568762), the Brinson Foundation, and the National Science Foundation (Grants No. PHY2011968, No. PHY-2011961, and No. PHY-1836809). V. V. is generously supported by a Klarman Fellowship at Cornell, the Sherman Fairchild Foundation, and NSF Grants No. PHY-170212 and No. PHY-1708213 at Caltech. The computations presented here were conducted on the Caltech High Performance Cluster, partially supported by a grant from the Gordon and Betty Moore Foundation.

\section{APPENDIX A: SpEC RUNS-SKu CONFIGURATION}

We summarize our NR simulations of SKu BBHs in Table V. We remark that the SKu condition is not well preserved after the junk-radiation regime. Nevertheless, the maximum recoil velocity $v_{f}^{z}$ is $4050 \mathrm{~km} \mathrm{~s}^{-1}$, and it is roughly proportional to $\chi_{\text {init }}$.

\section{APPENDIX B: BOB FOR $h_{22}$ AND $\ddot{h}_{22}$}

In this section, we discuss the BOB model for $h_{22}$ and $\ddot{h}_{22}$.

\section{1. $\ddot{h}_{22}$}

Let us start from $\ddot{h}_{22}$. As discussed in Ref. [111]

$$
\begin{aligned}
\ddot{h}_{22} & =\frac{d}{d t} \dot{h}_{22}=\frac{d}{d t}\left|\dot{h}_{22}\right| e^{-i \phi_{22}(t)} \sim-i \dot{\phi}_{22}\left|\dot{h}_{22}\right| e^{-i \phi_{22}(t)} \\
& =-i \Omega_{22}\left|\dot{h}_{22}\right| e^{-i \phi_{22}(t)}
\end{aligned}
$$

where we have assumed that $\left|\dot{h}_{22}\right|$ changes much slower than $\phi_{22}$. The above equation implies that the frequency of $\ddot{h}_{22}$ and $\dot{h}_{22}$ are roughly the same. Therefore, below we do not distinguish the frequency of $\ddot{h}_{22}$ from that of $\dot{h}_{22}$, and use $\Omega_{22}$ to stand for both frequencies. Combining Eqs. (44) with (B1), we obtain

$$
\left|\dot{h}_{22}\right|^{2} \sim\left|\ddot{h}_{22}\right|^{2} / \Omega_{22}^{2} \propto \frac{d}{d t} \Omega_{22}^{2} .
$$

Then applying Eq. (45) to $\left|\ddot{h}_{22}\right|{ }^{7}$ i.e.,

$$
\left|\ddot{h}_{22}\right|=X \operatorname{sech}\left[\gamma\left(t-t_{p}\right)\right],
$$

which leads to

$$
\Omega_{22}=\left\{\Omega_{0}^{4}+\frac{\omega_{0}^{4}-\Omega_{0}^{4}}{2}\left[\tanh \gamma\left(t-t_{p}\right)+1\right]\right\}^{1 / 4} .
$$

The above equation implies

$$
\lim _{t \rightarrow \infty} \Omega_{22}^{(2)}=\omega_{0},
$$

which is the same as the case of $\dot{h}_{22}$ [Eq. (48)]. Integrating Eq. (B4) again gives the time dependence of $\phi_{22}$, i.e., the phase of $\ddot{h}_{22}$

$$
\begin{aligned}
\phi_{22}= & \frac{1}{\gamma}\left\{\omega_{0}\left(\operatorname{arctanh} \frac{\Omega}{\omega_{0}}+\arctan \frac{\Omega}{\omega_{0}}\right)\right. \\
& \left.-\Omega_{0}\left(\operatorname{arccoth} \frac{\Omega_{0}}{\Omega}+\operatorname{arccot} \frac{\Omega_{0}}{\Omega}\right)\right\}-\phi_{0} .
\end{aligned}
$$

This is the original form of BOB model [cf. Eq. (10) of Ref. [111]]. Clearly, Eq. (B6) is different from Eq. (49).

\footnotetext{
${ }^{7}$ We use the same notation as Eq. (46) since this will not cause any confusion.
} 
TABLE V. A summary for SKu configurations. The convention is the same as the one used in Table I, except that the fifth and sixth columns are the components of individual spin in the Cartesian coordinates, where the $z$-axis is in the direction of orbital angular momentum; the line of two BHs determines the $x$-axis; and the right-handed rule determines the $y$-axis. The dimensionless spin ranges from 0.6 to 0.95 , specified at the orbital frequency $\Omega_{\text {orb }}$.

\begin{tabular}{|c|c|c|c|c|c|c|c|c|c|}
\hline \multicolumn{4}{|c|}{ Run label } & \multirow[b]{2}{*}{$\chi_{1}$} & \multirow[b]{2}{*}{$\chi_{2}$} & \multirow[b]{2}{*}{$\left|\chi_{1}\right|=\left|\chi_{2}\right|$} & \multirow[b]{2}{*}{$m_{f}$} & \multirow[b]{2}{*}{$\begin{array}{c}v_{f}^{z} \\
\left(\times 10^{-3}\right) \\
\end{array}$} & \multirow[b]{2}{*}{$\chi_{f}$} \\
\hline & $\begin{array}{l}\text { This } \\
\text { paper }\end{array}$ & $\begin{array}{l}\text { SXS: } \\
\text { BBH }\end{array}$ & $\begin{array}{c}\Omega_{\text {orb }} \\
\left(\times 10^{-2}\right)\end{array}$ & & & & & & \\
\hline \multirow[t]{8}{*}{$\overline{\mathrm{SKu} 6}$} & “01" & 2428 & 1.63 & $(0.378,-0.378,0.273)$ & $(-0.413,0.389,0.200)$ & 0.6 & 0.944 & -1.46 & 0.754 \\
\hline & “02” & 2429 & 1.62 & $(0.390,0.359,0.281)$ & $(-0.402,-0.398,0.199)$ & 0.6 & 0.942 & -8.04 & 0.749 \\
\hline & “03” & 2430 & 1.63 & $(-0.374,0.383,0.271)$ & $(0.406,-0.395,0.199)$ & 0.6 & 0.944 & -0.34 & 0.754 \\
\hline & “04” & 2431 & 1.62 & $(-0.386,-0.364,0.281)$ & $(0.397,0.402,0.201)$ & 0.6 & 0.942 & 8.03 & 0.749 \\
\hline & “05” & 2432 & 1.63 & $(0.254,0.465,0.282)$ & $(-0.254,-0.504,0.202)$ & 0.6 & 0.942 & -7.54 & 0.749 \\
\hline & “06” & 2448 & 1.63 & $(0.533,-0.0207,0.275)$ & $\left(-0.568,2.00 \times 10^{-3}, 0.193\right)$ & 0.6 & 0.944 & -6.80 & 0.752 \\
\hline & “07” & 2449 & 1.63 & $\left(-4.75 \times 10^{-3}, 0.531,0.279\right)$ & $(0.0218,-0.564,0.203)$ & 0.6 & 0.942 & -6.01 & 0.750 \\
\hline & $" 08 ”$ & 2450 & 1.63 & $(0.0120,-0.531,0.280)$ & $(-0.0312,0.564,0.202)$ & 0.6 & 0.942 & 5.91 & 0.750 \\
\hline \multirow[t]{4}{*}{ SKu8 } & “01” & 2433 & 1.63 & $(0.666,0.308,0.320)$ & $(-0.667,-0.314,0.311)$ & 0.8 & 0.941 & -1.58 & 0.773 \\
\hline & “02” & 2434 & 1.63 & $(-0.352,0.647,0.312)$ & $(0.360,-0.649,0.300)$ & 0.8 & 0.939 & -11.0 & 0.767 \\
\hline & $" 03 ”$ & 2435 & 1.63 & $(-0.669,-0.306,0.316)$ & $(0.667,0.309,0.316)$ & 0.8 & 0.941 & 1.31 & 0.773 \\
\hline & "04" & 2436 & 1.63 & $(0.382,-0.629,0.315)$ & $(-0.390,0.630,0.301)$ & 0.8 & 0.939 & 11.0 & 0.766 \\
\hline \multirow[t]{11}{*}{ SKu95 } & $" 01 ”$ & 2437 & 1.63 & $(-0.793,-0.437,0.284)$ & $(0.792,0.437,0.290)$ & 0.95 & 0.942 & 2.43 & 0.765 \\
\hline & “02” & 2438 & 1.62 & $(0.422,-0.803,0.280)$ & $(-0.423,0.804,0.279)$ & 0.95 & 0.938 & 13.5 & 0.752 \\
\hline & “03” & 2439 & 1.63 & $(0.800,0.423,0.288)$ & $(-0.800,-0.426,0.283)$ & 0.95 & 0.942 & -4.29 & 0.765 \\
\hline & “04” & 2440 & 1.63 & $(-0.428,0.802,0.277)$ & $(0.425,-0.801,0.283)$ & 0.95 & 0.938 & -13.5 & 0.753 \\
\hline & “05” & 2441 & 1.63 & $(-0.826,-0.377,0.277)$ & $(0.824,0.376,0.284)$ & 0.95 & 0.941 & 10.6 & 0.760 \\
\hline & “06” & 2442 & 1.62 & $(0.390,-0.821,0.275)$ & $(-0.390,0.822,0.2733)$ & 0.95 & 0.938 & 13.5 & 0.750 \\
\hline & “07” & 2443 & 1.62 & $(-0.358,0.837,0.272)$ & $(0.355,-0.836,0.278)$ & 0.95 & 0.938 & -13.5 & 0.751 \\
\hline & “08” & 2444 & 1.64 & $(0.293,-0.820,0.380)$ & $(-0.301,0.825,0.363)$ & 0.95 & 0.936 & -2.80 & 0.785 \\
\hline & “09” & 2445 & 1.65 & $(0.826,0.279,0.375)$ & $(-0.829,-0.281,0.368)$ & 0.95 & 0.933 & 13.0 & 0.776 \\
\hline & “10” & 2446 & 1.64 & $(-0.229,0.842,0.376)$ & $(0.239,-0.850,0.351)$ & 0.95 & 0.936 & 3.35 & 0.784 \\
\hline & "11" & 2447 & 1.65 & $(-0.836,-0.252,0.372)$ & $(0.837,0.251,0.372)$ & 0.95 & 0.933 & -12.4 & 0.776 \\
\hline
\end{tabular}

\section{2. $h_{22}$}

Following the same line of reasoning, the frequency of $h_{22}$ is also approximately equal to $\Omega_{22}$. Therefore

$$
\left|\dot{h}_{22}\right|^{2} \sim\left|h_{22}\right|^{2} \Omega_{22}^{2} \propto \frac{d}{d t} \Omega_{22}^{2} .
$$

Then using the assumption

$$
\left|h_{22}\right|=X \operatorname{sech}\left[\gamma\left(t-t_{p}\right)\right]
$$

we obtain

$$
\Omega_{22}=\Omega_{0} X \gamma^{-1 / 2}\left[\tanh \gamma\left(t-t_{p}\right)+1\right]^{1 / 2} .
$$

Integrating the above equation again can lead to a tedious expression of $\phi_{22}$, we do not show it here.

The BOB model for $h_{22}, \ddot{h}_{22}$, together with the one for $\dot{h}_{22}$ [Eq. (46)], are used to fit NR results, and are compared to QNMs in Fig. 17. We can see the model works the worst for $h_{22}$.

\section{APPENDIX C: THE PARITY TRANSFORMATION OF A COMPLEX STRAIN}

In this section, we show that the complex strain $h=$ $h_{+}-i h_{\times}$is transformed to the complex conjugate if the whole system undergoes a parity transformation (including the BBH system and observer).

According to Fig. 9, under the parity transformation two $\mathrm{BHs}$ exchange their locations, while have their individual spin fixed, since axial vectors are not changed by the parity transformation. Meanwhile, within the detector frame, the orientation of detector arms and the propagation direction are flipped simultaneously, as shown in Figs. 23(a) and 23(b). We want to emphasize that the $\mathrm{GW}$ detector is a 2D plane (formed by two arms). Its parity transformation can be equivalently achieved by a $\pi$-rotation about the axis that is perpendicular to the detector plane. Therefore, we further rotate the whole system about the 


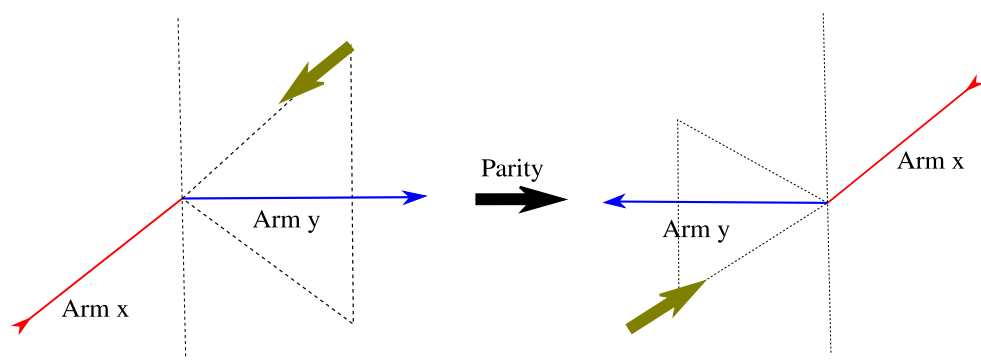

$\left(\theta_{S}, \phi_{S}\right)$

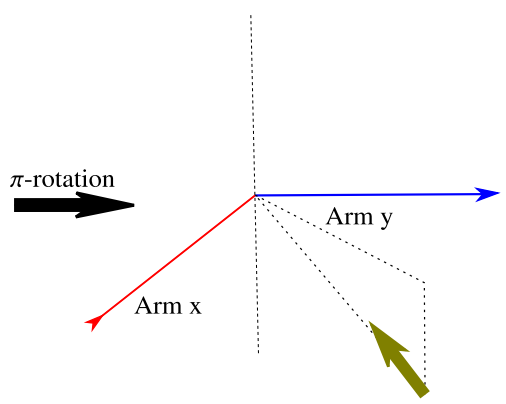

$\left(\pi-\theta_{S}, \phi_{S}\right)$

(c)

FIG. 23. Parity inversion of a SKd BBH binary system within the detector frame. The arrow stands for the direction of incoming GW. The system undergoes a parity inversion from (a) to (b). We further rotate the whole system around the vertical dash line by $\pi$, which leads to (c). Comparing (a) and (c), the polar angle of sky location $\theta_{S}$ becomes supplementary under the transformation.

vertical dashed line by $\pi$, as shown in Fig. 23(c). We can see that the detector configuration changes back to the one of (a), while the sky location of GW source changes from $\left(\theta_{S}, \phi_{S}\right)$ (the northern hemisphere) to $\left(\pi-\theta_{S}, \phi_{S}\right)$ (the southern hemisphere).

Since GR preserves the parity, the strain $h_{\mathrm{obs}}$ observed by a detector:

$$
h_{\mathrm{obs}}=h_{+} F_{+}+h_{\times} F_{\times},
$$

is not affected by the above mentioned transformations. The antenna patterns $F_{+, x}$ have forms [127]

$$
F_{+}=\frac{1}{2}\left(1+\cos ^{2} \theta_{S}\right) \cos 2 \phi_{S}, \quad F_{\times}=\cos \theta_{S} \sin 2 \phi_{S},
$$

with $\left(\theta_{S}, \phi_{S}\right)$ the sky location of GW source relative to the detector. Under the transformation from Figs. 23(a) to 23(c), i.e., $\left(\theta_{S}, \phi_{S}\right) \rightarrow\left(\pi-\theta_{S}, \phi_{S}\right)$ the antenna patterns $F_{+, \times}$transform as

$$
F_{+} \rightarrow F_{+}, \quad F_{\times} \rightarrow-F_{\times} .
$$

Recalling that the $h_{\text {obs }}$ of Figs. 23(a) and 23(c) are the same, we then have

$$
h_{+} \rightarrow h_{+}, \quad h_{\times} \rightarrow-h_{\times} .
$$

As a result,

$$
h=h_{+}-i h_{\times} \rightarrow h^{*}=h_{+}+i h_{\times} .
$$

[1] R. Abbott et al. (LIGO Scientific, Virgo Collaborations), Phys. Rev. Lett. 125, 101102 (2020).

[2] R. Abbott et al. (LIGO Scientific, Virgo Collaborations), Astrophys. J. Lett. 900, L13 (2020).

[3] M. Graham et al., Phys. Rev. Lett. 124, 251102 (2020).

[4] S. E. Woosley, Astrophys. J. 836, 244 (2017).

[5] S. E. Woosley, Astrophys. J. 878, 49 (2019).

[6] V. Gayathri, J. Healy, J. Lange, B. O'Brien, M. Szczepanczyk, I. Bartos, M. Campanelli, S. Klimenko, C. Lousto, and R. O'Shaughnessy, arXiv:2009.05461.

[7] R. Gamba, M. Breschi, G. Carullo, P. Rettegno, S. Albanesi, S. Bernuzzi, and A. Nagar, arXiv:2106 .05575 .

[8] J. C. Bustillo, N. Sanchis-Gual, A. Torres-Forné, and J. A. Font, Phys. Rev. Lett. 126, 201101 (2021).

[9] J. C. Bustillo, N. Sanchis-Gual, A. Torres-Forné, J. A. Font, A. Vajpeyi, R. Smith, C. Herdeiro, E. Radu, and S. H. W. Leong, Phys. Rev. Lett. 126, 081101 (2021).
[10] J. Aasi et al. (LIGO Scientific Collaboration), Classical Quantum Gravity 32, 074001 (2015).

[11] F. Acernese et al. (VIRGO Collaboration), Classical Quantum Gravity 32, 024001 (2015).

[12] T. Akutsu et al. (KAGRA Collaboration), Nat. Astron. 3, 35 (2019).

[13] T. Akutsu et al. (KAGRA Collaboration), arXiv:2005 .05574

[14] S. Biscoveanu, M. Isi, V. Varma, and S. Vitale, arXiv: 2106.06492.

[15] K. D. Kokkotas and B. G. Schmidt, Living Rev. Relativity 2, 2 (1999).

[16] B. Carter, Phys. Rev. Lett. 26, 331 (1971).

[17] O. Dreyer, B. J. Kelly, B. Krishnan, L. S. Finn, D. Garrison, and R. Lopez-Aleman, Classical Quantum Gravity 21, 787 (2004).

[18] E. Berti, V. Cardoso, and C. M. Will, Phys. Rev. D 73, 064030 (2006). 
[19] E. Berti, J. Cardoso, V. Cardoso, and M. Cavaglia, Phys. Rev. D 76, 104044 (2007).

[20] S. Gossan, J. Veitch, and B. Sathyaprakash, Phys. Rev. D 85, 124056 (2012).

[21] J. Meidam, M. Agathos, C. Van Den Broeck, J. Veitch, and B. Sathyaprakash, Phys. Rev. D 90, 064009 (2014).

[22] E. Berti et al., Classical Quantum Gravity 32, 243001 (2015).

[23] E. Berti, A. Sesana, E. Barausse, V. Cardoso, and K. Belczynski, Phys. Rev. Lett. 117, 101102 (2016).

[24] V. Baibhav, E. Berti, V. Cardoso, and G. Khanna, Phys. Rev. D 97, 044048 (2018).

[25] V. Baibhav and E. Berti, Phys. Rev. D 99, 024005 (2019).

[26] E. Berti, K. Yagi, H. Yang, and N. Yunes, Gen. Relativ. Gravit. 50, 49 (2018).

[27] R. Brito, A. Buonanno, and V. Raymond, Phys. Rev. D 98, 084038 (2018).

[28] G. Carullo et al., Phys. Rev. D 98, 104020 (2018).

[29] M. Isi, M. Giesler, W. M. Farr, M. A. Scheel, and S. A. Teukolsky, Phys. Rev. Lett. 123, 111102 (2019).

[30] M. Giesler, M. Isi, M. A. Scheel, and S. Teukolsky, Phys. Rev. X 9, 041060 (2019).

[31] C. D. Capano, M. Cabero, J. Abedi, S. Kastha, J. Westerweck, A. H. Nitz, A. B. Nielsen, and B. Krishnan, arXiv:2105.05238.

[32] B. Abbott et al. (LIGO Scientific, Virgo Collaborations), Phys. Rev. D 100, 104036 (2019).

[33] B. Abbott et al. (LIGO Scientific, Virgo Collaborations), Phys. Rev. Lett. 116, 221101 (2016); 121, 129902(E) (2018).

[34] G. Carullo, W. Del Pozzo, and J. Veitch, Phys. Rev. D 99, 123029 (2019); 100, 089903(E) (2019).

[35] M. Cabero, C. D. Capano, O. Fischer-Birnholtz, B. Krishnan, A. B. Nielsen, A. H. Nitz, and C. M. Biwer, Phys. Rev. D 97, 124069 (2018).

[36] W. Del Pozzo and A. Nagar, Phys. Rev. D 95, 124034 (2017).

[37] V. Cardoso, M. Kimura, A. Maselli, E. Berti, C. F. Macedo, and R. McManus, Phys. Rev. D 99, 104077 (2019).

[38] R. McManus, E. Berti, C. F. Macedo, M. Kimura, A. Maselli, and V. Cardoso, Phys. Rev. D 100, 044061 (2019).

[39] A. Maselli, P. Pani, L. Gualtieri, and E. Berti, Phys. Rev. D 101, 024043 (2020).

[40] L. Rezzolla, E. N. Dorband, C. Reisswig, P. Diener, D. Pollney, E. Schnetter, and B. Szilagyi, Astrophys. J. 679, 1422 (2008).

[41] L. Rezzolla, E. Barausse, E. N. Dorband, D. Pollney, C. Reisswig, J. Seiler, and S. Husa, Phys. Rev. D 78, 044002 (2008).

[42] L. Rezzolla, P. Diener, E. N. Dorband, D. Pollney, C. Reisswig, E. Schnetter, and J. Seiler, Astrophys. J. Lett. 674, L29 (2008).

[43] A. Buonanno, L. E. Kidder, and L. Lehner, Phys. Rev. D 77, 026004 (2008).

[44] W. Tichy and P. Marronetti, Phys. Rev. D 78, 081501 (2008).

[45] M. Kesden, Phys. Rev. D 78, 084030 (2008).

[46] E. Barausse and L. Rezzolla, Astrophys. J. Lett. 704, L40 (2009).

[47] M. Kesden, U. Sperhake, and E. Berti, Phys. Rev. D 81, 084054 (2010).
[48] E. Barausse, V. Morozova, and L. Rezzolla, Astrophys. J. 758, 63 (2012); 786, 76(E) (2014).

[49] J. Healy, C. O. Lousto, and Y. Zlochower, Phys. Rev. D 90, 104004 (2014).

[50] J. Healy and C. O. Lousto, Phys. Rev. D 95, 024037 (2017).

[51] X. Jiménez-Forteza, D. Keitel, S. Husa, M. Hannam, S. Khan, and M. Pürrer, Phys. Rev. D 95, 064024 (2017).

[52] F. Hofmann, E. Barausse, and L. Rezzolla, Astrophys. J. Lett. 825, L19 (2016).

[53] J. Healy and C. O. Lousto, Phys. Rev. D 97, 084002 (2018).

[54] V. Varma, D. Gerosa, L. C. Stein, F. Hébert, and H. Zhang, Phys. Rev. Lett. 122, 011101 (2019).

[55] V. Varma, S. E. Field, M. A. Scheel, J. Blackman, D. Gerosa, L. C. Stein, L. E. Kidder, and H. P. Pfeiffer, Phys. Rev. Research 1, 033015 (2019).

[56] D. Ferguson, S. Ghonge, J. A. Clark, J. C. Bustillo, P. Laguna, D. Shoemaker, and J. C. Bustillo, Phys. Rev. Lett. 123, 151101 (2019).

[57] T. Damour and A. Nagar, Phys. Rev. D 76, 044003 (2007).

[58] L. Reali, M. Mould, D. Gerosa, and V. Varma, Classical Quantum Gravity 37, 225005 (2020).

[59] J. Healy, P. Laguna, and D. Shoemaker, Classical Quantum Gravity 31, 212001 (2014).

[60] A. Taylor and V. Varma, Phys. Rev. D 102, 104047 (2020).

[61] J. D. Bekenstein, Astrophys. J. 183, 657 (1973).

[62] A. Peres, Phys. Rev. 128, 2471 (1962).

[63] W. B. Bonnor and M. A. Rotenberg, Proc. R. Soc. A 265, 109 (1961).

[64] A. G. Wiseman, Phys. Rev. D 46, 1517 (1992).

[65] L. E. Kidder, Phys. Rev. D 52, 821 (1995).

[66] L. Blanchet, M. S. Qusailah, and C. M. Will, Astrophys. J. 635, 508 (2005).

[67] J. D. Schnittman and A. Buonanno, Astrophys. J. Lett. 662, L63 (2007).

[68] C. F. Sopuerta, N. Yunes, and P. Laguna, Phys. Rev. D 74, 124010 (2006); 75, 069903(E) (2007); 78, 049901(E) (2008).

[69] B. Bruegmann, J. A. Gonzalez, M. Hannam, S. Husa, and U. Sperhake, Phys. Rev. D 77, 124047 (2008).

[70] J. D. Schnittman, A. Buonanno, J. R. van Meter, J. G. Baker, W. D. Boggs, J. Centrella, B. J. Kelly, and S. T. McWilliams, Phys. Rev. D 77, 044031 (2008).

[71] K. S. Thorne, Rev. Mod. Phys. 52, 299 (1980).

[72] J. A. Gonzalez, U. Sperhake, B. Bruegmann, M. Hannam, and S. Husa, Phys. Rev. Lett. 98, 091101 (2007).

[73] F. Herrmann, I. Hinder, D. Shoemaker, and P. Laguna, Classical Quantum Gravity 24, S33 (2007).

[74] J. G. Baker, J. Centrella, D.-I. Choi, M. Koppitz, J. R. van Meter, and M. Miller, Astrophys. J. Lett. 653, L93 (2006).

[75] C. O. Lousto and Y. Zlochower, Phys. Rev. D 77, 044028 (2008).

[76] J. G. Baker, W. D. Boggs, J. Centrella, B. J. Kelly, S. T. McWilliams, M. Miller, and J. R. van Meter, Astrophys. J. 668, 1140 (2007).

[77] M. Koppitz, D. Pollney, C. Reisswig, L. Rezzolla, J. Thornburg, P. Diener, and E. Schnetter, Phys. Rev. Lett. 99, 041102 (2007).

[78] J. Gonzalez, M. Hannam, U. Sperhake, B. Bruegmann, and S. Husa, Phys. Rev. Lett. 98, 231101 (2007). 
[79] M. Campanelli, C. O. Lousto, Y. Zlochower, and D. Merritt, Astrophys. J. Lett. 659, L5 (2007).

[80] M. Campanelli, C. O. Lousto, Y. Zlochower, and D. Merritt, Phys. Rev. Lett. 98, 231102 (2007).

[81] F. Herrmann, I. Hinder, D. Shoemaker, P. Laguna, and R. A. Matzner, Astrophys. J. 661, 430 (2007).

[82] W. Tichy and P. Marronetti, Phys. Rev. D 76, 061502 (2007).

[83] F. Herrmann, I. Hinder, D. M. Shoemaker, P. Laguna, and R. A. Matzner, Phys. Rev. D 76, 084032 (2007).

[84] M. Campanelli, C. Lousto, and Y. Zlochower, Phys. Rev. D 74, 041501 (2006).

[85] C. O. Lousto and Y. Zlochower, Phys. Rev. Lett. 107, 231102 (2011).

[86] C. O. Lousto and J. Healy, Phys. Rev. D 100, 104039 (2019).

[87] F. Pretorius, arXiv:0710.1338.

[88] D. Keppel, D. A. Nichols, Y. Chen, and K. S. Thorne, Phys. Rev. D 80, 124015 (2009).

[89] G. Lovelace, Y. Chen, M. Cohen, J. D. Kaplan, D. Keppel, K. D. Matthews, D. A. Nichols, M. A. Scheel, and U. Sperhake, Phys. Rev. D 82, 064031 (2010).

[90] S. E. Gralla and F. Herrmann, Classical Quantum Gravity 30, 205009 (2013).

[91] D. Merritt, M. Milosavljevic, M. Favata, S. A. Hughes, and D. E. Holz, Astrophys. J. Lett. 607, L9 (2004).

[92] E. Bonning, G. Shields, and S. Salviander, Astrophys. J. Lett. 666, L13 (2007).

[93] M. Volonteri, Astrophys. J. Lett. 663, L5 (2007).

[94] S. Komossa, Adv. Astron. 2012, 364973 (2012).

[95] K. Chamberlain, C. J. Moore, D. Gerosa, and N. Yunes, Phys. Rev. D 99, 024025 (2019).

[96] D. Gerosa and C. J. Moore, Phys. Rev. Lett. 117, 011101 (2016).

[97] V. Varma, M. Isi, and S. Biscoveanu, Phys. Rev. Lett. 124, 101104 (2020).

[98] M. Kesden, U. Sperhake, and E. Berti, Astrophys. J. 715, 1006 (2010).

[99] J. Blackman, S. E. Field, C. R. Galley, B. Szilágyi, M. A. Scheel, M. Tiglio, and D. A. Hemberger, Phys. Rev. Lett. 115, 121102 (2015).

[100] J. Blackman, S. E. Field, M. A. Scheel, C. R. Galley, D. A. Hemberger, P. Schmidt, and R. Smith, Phys. Rev. D 95, 104023 (2017).

[101] J. Blackman, S. E. Field, M. A. Scheel, C. R. Galley, C. D. Ott, M. Boyle, L. E. Kidder, H. P. Pfeiffer, and B. Szilágyi, Phys. Rev. D 96, 024058 (2017).
[102] V. Varma, S. E. Field, M. A. Scheel, J. Blackman, L. E. Kidder, and H. P. Pfeiffer, Phys. Rev. D 99, 064045 (2019).

[103] D. Gerosa, F. Hébert, and L. C. Stein, Phys. Rev. D 97, 104049 (2018).

[104] H. Yu, S. Ma, M. Giesler, and Y. Chen, Phys. Rev. D 102, 123009 (2020).

[105] S. A. Hughes, A. Apte, G. Khanna, and H. Lim, Phys. Rev. Lett. 123, 161101 (2019).

[106] A. Apte and S. A. Hughes, Phys. Rev. D 100, 084031 (2019).

[107] H. Lim, G. Khanna, A. Apte, and S. A. Hughes, Phys. Rev. D 100, 084032 (2019).

[108] https://www.black-holes.org/code/spec.html.

[109] M. Boyle et al., Classical Quantum Gravity 36, 195006 (2019).

[110] A. H. Mroue et al., Phys. Rev. Lett. 111, 241104 (2013).

[111] S. T. McWilliams, Phys. Rev. Lett. 122, 191102 (2019).

[112] C. W. Misner, K. S. Thorne, and J. A. Wheeler, Gravitation (MacMillan, London, 1973).

[113] W. H. Press and S. A. Teukolsky, Astrophys. J. 185, 649 (1973).

[114] E. Berti and A. Klein, Phys. Rev. D 90, 064012 (2014).

[115] L. C. Stein, J. Open Source Software 4, 1683 (2019).

[116] E. Finch and C. J. Moore, Phys. Rev. D 103, 084048 (2021).

[117] M. Ruiz, R. Takahashi, M. Alcubierre, and D. Nunez, Gen. Relativ. Gravit. 40, 2467 (2008).

[118] R. A. Porto, A. Ross, and I. Z. Rothstein, J. Cosmol. Astropart. Phys. 03 (2011) 009.

[119] I. Kamaretsos, M. Hannam, S. Husa, and B. Sathyaprakash, Phys. Rev. D 85, 024018 (2012).

[120] I. Kamaretsos, M. Hannam, and B. Sathyaprakash, Phys. Rev. Lett. 109, 141102 (2012).

[121] D. A. Nichols and Y. Chen, Phys. Rev. D 85, 044035 (2012).

[122] J. G. Baker, W. D. Boggs, J. Centrella, B. J. Kelly, S. T. McWilliams, and J. R. van Meter, Phys. Rev. D 78, 044046 (2008).

[123] B. P. Abbott et al. (LIGO Scientific Collaboration), Classical Quantum Gravity 34, 044001 (2017).

[124] L. London, D. Shoemaker, and J. Healy, Phys. Rev. D 90, 124032 (2014); 94, 069902(E) (2016).

[125] V. Baibhav, E. Berti, and V. Cardoso, Phys. Rev. D 101, 084053 (2020).

[126] E. Racine, Phys. Rev. D 78, 044021 (2008).

[127] E. Poisson and C. M. Will, Gravity: Newtonian, PostNewtonian, Relativistic (Cambridge University Press, Cambridge, England, 2014). 